\title{
Pacific
}

Journal of

Mathematics

\section{GROUP GRADINGS ON \\ RESTRICTED CARTAN-TYPE LIE ALGEBRAS}

YURi BAHTURIN AND MikHaIL Kochetov 


\title{
GROUP GRADINGS ON RESTRICTED CARTAN-TYPE LIE ALGEBRAS
}

\author{
YURI BAHTURIN AND MIKHAIL KOCHETOV
}

For a given abelian group $G$, we classify the isomorphism classes of $G$ gradings on the simple restricted Lie algebras of types $W(m ; 1)$ and $S(m ; \underline{1})$ for $m \geq 2$, in terms of numerical and group-theoretical invariants. Our main tool is automorphism group schemes, which we determine for the simple restricted Lie algebras of types $S(m ; \underline{1})$ and $H(m ; \underline{1})$. The ground field is assumed to be algebraically closed of characteristic $p>3$.

\section{Introduction}

Let $U$ be an algebra (not necessarily associative) over a field $\mathbb{F}$ and let $G$ be a group, written multiplicatively.

Definition 1.1. A $G$-grading on $U$ is a vector space decomposition

$$
U=\bigoplus_{g \in G} U_{g}
$$

such that

$$
U_{g} U_{h} \subset U_{g h} \text { for all } g, h \in G .
$$

The subspace $U_{g}$ is called the homogeneous component of degree $g$. The support of the $G$-grading is the set

$$
\left\{g \in G \mid U_{g} \neq 0\right\} .
$$

If $U$ is finite-dimensional, then, replacing $G$ with the subgroup generated by the support of the grading, we may assume without loss of generality that $G$ is finitely generated.

Definition 1.2. Two $G$-gradings $U=\bigoplus_{g \in G} U_{g}$ and $U=\bigoplus_{g \in G} U_{g}^{\prime}$ are isomorphic if there exists an algebra automorphism $\psi: U \rightarrow U$ such that

$$
\psi\left(U_{g}\right)=U_{g}^{\prime} \quad \text { for all } g \in G,
$$

that is, if $U=\bigoplus_{g \in G} U_{g}$ and $U=\bigoplus_{g \in G} U_{g}^{\prime}$ are isomorphic as $G$-graded algebras.

Bahturin acknowledges support by NSERC grant number 227060-04. Kochetov acknowledges support by NSERC Discovery Grant number 341792-07.

MSC2000: primary 17B70; secondary 17B60.

Keywords: graded algebra, simple Lie algebra, grading, Cartan-type Lie algebra. 
Since the same vector space decomposition can be regarded as a $G$-grading for different groups $G$, one can also define equivalence of gradings, which is a weaker relation than isomorphism - see, for example, [Kochetov 2009] for a discussion. Fine gradings (that is, those that cannot be refined) as well as their universal groups are of particular interest.

We are interested in the problem of finding all possible group gradings on finitedimensional simple Lie algebras. If $L$ is a simple Lie algebra, then it is known that the support of any $G$-grading on $L$ generates an abelian group. Hence, in this paper we will always assume that $G$ is abelian. We will also assume that the ground field $\mathbb{F}$ is algebraically closed.

For an arbitrary abelian group $G$, the classification of $G$-gradings (up to isomorphism) is known for almost all classical simple Lie algebras over an algebraically closed field of characteristic 0 or $p>2$. The classification of fine gradings (up to equivalence) is also known. Refer to [Bahturin and Kochetov 2010; Elduque 2010] and references therein.

Our goal is to carry out the same classification for simple restricted Lie algebras of Cartan type. In the present paper, we achieve this goal in characteristic $p>3$ for Witt algebras in Theorem 4.13 and Corollary 4.14 and for special algebras (see the definitions in Section 2) in Theorem 4.17 and Corollary 4.18.

In a number of cases, a fruitful approach to the classification of gradings by abelian groups on an algebra $U$ is to use another algebra, $R$, that shares with $U$ the automorphism group scheme (see Section 3 ) and whose gradings are easier to study. This approach often requires equipping $R$ with some additional structure. For classical simple Lie algebras of series $A, B, C$ and $D$, one takes for $R$ the matrix algebra $M_{n}(\mathbb{F})$, possibly equipped with an antiautomorphism.

For Lie algebras of Cartan type, $R$ is the "coordinate algebra" $O(m ; \underline{n})$ (see Definition 2.1). In the restricted case, this is just the truncated polynomial algebra $\mathcal{O}(m ; \underline{1})=\mathbb{F}\left[x_{1}, \ldots, x_{m}\right] /\left(x_{1}^{p}, \ldots, x_{m}^{p}\right)$. It is not difficult to classify $G$-gradings on the algebra $\mathcal{O}(m ; \underline{1})$ - see Theorem 4.8 and Corollary 4.11 . Since $\mathcal{O}(m ; \underline{1})$ has the same automorphism group scheme as the Witt algebra $W(m ; \underline{1})$, this immediately gives the classification of gradings for the latter. In Section 3, we show that the automorphism group scheme of the special algebra $S(m ; \underline{1})^{(1)}$ for $m \geq 3$, respectively Hamiltonian algebra $H(m ; \underline{1})^{(2)}$ for $m=2 r$, is isomorphic to the stabilizer of the differential form $\omega_{S}$, respectively $\omega_{H}$, in the automorphism group scheme of $\mathcal{O}(m ; 1)$ - see Theorems 3.2 and 3.5 , respectively. As a consequence, all gradings on $S(m ; \underline{1})^{(1)}$ and $H(m ; \underline{1})^{(2)}$ come from gradings on $\mathcal{O}(m ; \underline{1})$. However, this does not yet give a classification of gradings on $S(m ; \underline{1})^{(1)}$ and $H(m ; \underline{1})^{(2)}$ up to isomorphism, because one must make sure that the isomorphism preserves the appropriate differential form - see Corollary 4.2. We then obtain a classification of gradings on $S(m ; \underline{1})^{(1)}$ for $m \geq 3$ and $S(2 ; \underline{1})^{(2)}=H(2 ; \underline{1})^{(2)}$ - see Theorem 4.17. The 
classification for $H(2 r ; \underline{1})^{(2)}$ with $r>1$ remains open.

The paper is structured as follows. In Section 2, we recall the definitions and basic facts regarding Lie algebras of Cartan type. In Section 3, we briefly recall background information on automorphism group schemes and determine them for $S(m ; \underline{1})^{(1)}$ and $H(m ; \underline{1})^{(2)}$. In Section 4 , we obtain the classification of gradings for Witt and special algebras (using the results on automorphism group schemes).

\section{Cartan-type Lie algebras}

We start by briefly recalling the definitions and relevant properties of Cartan-type Lie algebras. We will use [Strade 2004] as a standard reference. Fix $m \geq 1$ and $\underline{n}=\left(n_{1}, \ldots, n_{m}\right)$ where $n_{i} \geq 1$. Set

$$
\mathbb{Z}^{(m ; \underline{n})}:=\left\{\alpha \in \mathbb{Z}^{m} \mid 0 \leq \alpha_{i}<p^{n_{i}} \text { for } i=1, \ldots, m\right\} .
$$

The elements of $\mathbb{Z}^{(m ; \underline{n})}$ will be called multi-indices and denoted by Greek letters $\alpha, \beta, \gamma$. For $\alpha=\left(\alpha_{1}, \ldots, \alpha_{m}\right)$, set

$$
|\alpha|=\alpha_{1}+\cdots+\alpha_{m} .
$$

Denote by 1 the multi-index that has 1 in all positions and by $\varepsilon_{i}$ the multi-index that has 1 in position $i$ and zeros elsewhere.

Let $\mathbb{F}$ be a field of characteristic $p>0$.

Definition 2.1. The algebra $\mathbb{O}=\mathbb{O}(m ; \underline{n})$ over $\mathbb{F}$ is a commutative associative algebra with a basis $\left\{x^{(\alpha)} \mid \alpha \in \mathbb{Z}^{(m, \underline{n})}\right\}$ where multiplication given by

$$
x^{(\alpha)} x^{(\beta)}=\left(\begin{array}{c}
\alpha+\beta \\
\alpha
\end{array}\right) x^{(\alpha+\beta)} \quad \text { where }\left(\begin{array}{c}
\alpha+\beta \\
\alpha
\end{array}\right)=\prod_{i=1}^{m}\left(\begin{array}{c}
\alpha_{i}+\beta_{i} \\
\alpha_{i}
\end{array}\right) .
$$

If $\underline{n}=\underline{1}$, then $\mathbb{O} \cong \mathbb{F}\left[x_{1}, \ldots, x_{m}\right] /\left(x_{1}^{p}, \ldots, x_{m}^{p}\right)$ by identifying $x_{i}$ with $x^{\left(\varepsilon_{i}\right)}$ :

$$
x^{(\alpha)}=\frac{1}{\alpha_{1} ! \cdots \alpha_{m} !} x_{1}^{\alpha_{1}} \cdots x_{m}^{\alpha_{m}} .
$$

The algebra 0 has a canonical $\mathbb{Z}$-grading $O=\bigoplus_{\ell \geq 0} O_{\ell}$ defined by declaring the degree of $x^{(\alpha)}$ to be $|\alpha|$. The associated filtration will be denoted by

$$
\mathrm{O}_{(\ell)}:=\bigoplus_{j \geq \ell} \mathrm{O}_{j}
$$

Note that $\mathfrak{M}:=\mathrm{O}_{(1)}$ is the unique maximal ideal of $\mathrm{O}$.

We now define Witt, special and Hamiltonian graded Cartan-type Lie algebras. Contact algebras and generalized (that is, nongraded) Cartan-type Lie algebras will not be considered in this paper. 
Definition 2.2. Define a linear map $\partial_{i}: \mathcal{O} \rightarrow \mathcal{O}$ by $\partial_{i} x^{(\alpha)}=x^{\left(\alpha-\varepsilon_{i}\right)}$, where the righthand side is understood to be zero if $\alpha_{i}=0$. Then $\partial_{i}$ is a derivation of 0 . The Witt algebra $W=W(m ; \underline{n})$ is the subalgebra of $\operatorname{Der}(\mathcal{O})$ that consists of all operators of the form

$$
f_{1} \partial_{1}+\cdots+f_{m} \partial_{m} \quad \text { where } f_{i} \in \mathcal{O} .
$$

The canonical $\mathbb{Z}$-grading of 0 induces a $\mathbb{Z}$-grading on $\operatorname{End}(\mathcal{O})$. Since $W$ is a graded subspace of End(O), it inherits the $\mathbb{Z}$-grading: $W=\bigoplus_{\ell \geq-1} W_{\ell}$. Denote the associated filtration by $W_{(\ell)}$.

The de Rham complex

$$
\Omega^{0} \stackrel{d}{\longrightarrow} \Omega^{1} \stackrel{d}{\longrightarrow} \Omega^{2} \stackrel{d}{\longrightarrow} \cdots
$$

is defined as follows: $\Omega^{0}=\mathbb{O}, \Omega^{1}=\operatorname{Hom}_{\mathscr{O}}(W, \mathcal{O})$, and $\Omega^{k}=\left(\Omega^{1}\right)^{\wedge k}$ for $k \geq 2$. The map $d: \Omega^{0} \rightarrow \Omega^{1}$ is defined by $(d f)(D)=D(f)$ for all $f \in \mathbb{O}$ and $D \in W$. The remaining maps $d: \Omega^{k} \rightarrow \Omega^{k+1}$ are defined in the usual way: $d\left(f d x_{i_{1}} \wedge \cdots \wedge d x_{i_{k}}\right)=$ $d f \wedge d x_{i_{1}} \wedge \cdots \wedge d x_{i_{k}}$.

Any element $D \in W$ acts on $\Omega^{1}=\operatorname{Hom}_{\mathscr{O}}(W, \mathcal{O})$ by setting

$$
D(\omega)(E)=D(\omega(E))-\omega([D, E]) \quad \text { for all } \omega \in \Omega^{1} \text { and } E \in W .
$$

This action turns all the $\Omega^{k}=\left(\Omega^{1}\right)^{\wedge k}$ into $W$-modules. Of course, they also have canonical $\mathbb{Z}$-gradings and associated filtrations.

We will need the following differential forms to define the special and Hamiltonian algebras:

$$
\begin{aligned}
\omega_{S}:=d x_{1} \wedge d x_{2} \wedge \cdots \wedge d x_{m} \in \Omega^{m} & \text { if } m \geq 2, \\
\omega_{H}:=d x_{1} \wedge d x_{r+1}+d x_{2} \wedge d x_{r+2}+\cdots+d x_{r} \wedge d x_{2 r} \in \Omega^{2} & \text { if } m=2 r .
\end{aligned}
$$

Definition 2.3. The special algebra $S=S(m ; \underline{n})$ is the stabilizer of $\omega_{S}$ in $W(m ; \underline{n})$ :

$$
S=\left\{D \in W \mid D\left(\omega_{S}\right)=0\right\} .
$$

The Hamiltonian algebra $H=H(m ; \underline{n})$ is the stabilizer of $\omega_{H}$ in $W(m ; \underline{n})$ :

$$
H=\left\{D \in W \mid D\left(\omega_{H}\right)=0\right\} .
$$

In the case $m=2$, we have $\omega_{S}=\omega_{H}$ and hence $S=H$. It is well-known that $W(m ; \underline{n})$ is simple unless $p=2$ and $m=1$. The algebras $S(m ; \underline{n})$ and $H(m ; \underline{n})$ are not simple, but the first derived algebra $S(m ; \underline{n})^{(1)}$ for $m \geq 3$ and the second derived algebra $H(m ; \underline{n})^{(2)}$ are simple.

The Lie algebras $W(m ; \underline{n}), S(m ; \underline{n})$ and $H(m ; \underline{n})$ are restrictable - that is, admit $p$-maps making them restricted Lie algebras - if and only if $\underline{n}=\underline{1}$. From now on, we will assume that this is the case. Since $W, S$ and $H$ have trivial center, their $p$-maps are unique. Also, in this case $W=\operatorname{Der}(\mathcal{O})$ and hence the $p$-map is just the 
$p$-th power in the associative algebra $\operatorname{End}(0)$. The algebras $S$ and $H$ are restricted subalgebras of $W$.

\section{Automorphism group schemes}

Let $\mathcal{O}=\mathcal{O}(m ; 1)$. Any automorphism $\mu$ of the algebra $\mathcal{O}$ gives rise to an automorphism $\operatorname{Ad}(\mu)$ of $W$ given by $\operatorname{Ad}(\mu)(D)=\mu \circ D \circ \mu^{-1}$. Then we can define the action of $\mu$ on $\Omega^{1}=\operatorname{Hom}_{\mathscr{O}}(W, \mathcal{O})$ by setting

$$
\mu(\omega)(D)=\mu\left(\omega\left(\operatorname{Ad}\left(\mu^{-1}\right)(D)\right)\right)
$$

for all $\omega \in \Omega^{1}$ and $D \in W$. This turns all the $\Omega^{k}=\left(\Omega^{1}\right)^{\wedge k}$ into Aut(O)-modules. Clearly, these actions can still be defined in the same way if we extend the scalars from the base field $\mathbb{F}$ to any commutative associative $\mathbb{F}$-algebra $K$, that is, replace $\mathbb{O}$ with $\mathcal{O}(K):=\mathbb{O} \otimes K, W$ with $W(K):=W \otimes K$ and $\Omega^{k}$ with $\Omega^{k}(K):=\Omega^{k} \otimes K$.

Recall the automorphism group scheme of a finite-dimensional $\mathbb{F}$-algebra $U$ (see, for example, [Waterhouse 1979] for background on affine group schemes). As a functor, the (affine) group scheme $\operatorname{Aut}(U)$ is defined by setting $\operatorname{Aut}(U)(K)=$ $\operatorname{Aut}_{K}(U \otimes K)$ for any commutative associative $\mathbb{F}$-algebra $K$. From the discussion above it follows that we have morphisms of group schemes $\operatorname{Ad}: \operatorname{Aut}(0) \rightarrow \operatorname{Aut}(W)$ and also Aut $(\mathcal{O}) \rightarrow \mathrm{GL}\left(\Omega^{k}\right)$. (We identify a smooth algebraic group scheme such as $\mathrm{GL}(V)$ with the corresponding algebraic group.) Note that, since the $p$-map of $W \otimes K$ is uniquely determined, the automorphism group scheme of $W$ as a Lie algebra is the same as its automorphism group scheme as a restricted Lie algebra. Note also that the maps $d: \Omega^{k} \rightarrow \Omega^{k+1}$ are Aut(O)-equivariant.

The algebraic group scheme $\operatorname{Aut}(U)$ contains the algebraic group $\operatorname{Aut}(U)$ as the largest smooth subgroupscheme. The tangent Lie algebra of $\operatorname{Aut}(U)$ is $\operatorname{Der}(U)$, so $\operatorname{Aut}(U)$ is smooth if and only if $\operatorname{Der}(U)$ equals the tangent Lie algebra of the group $\operatorname{Aut}(U)$. The automorphism group schemes of simple Cartan-type Lie algebras, unlike those of the classical simple Lie algebras, are not smooth. Indeed, the tangent Lie algebra of Aut $(0)$ is $W_{(1)}$, which is a proper subalgebra of $W=\operatorname{Der}(0)$, so $\operatorname{Aut}(0)$ is not smooth. In view of the following theorem, we see that $\operatorname{Aut}(W)$ is not smooth.

Theorem 3.1 [Waterhouse 1971]. Let $\mathbb{O}=\mathbb{O}(m ; \underline{1})$ and $W=W(m ; \underline{1})$. Assume $p>3$. Then the morphism $\operatorname{Ad}: \operatorname{Aut}(0) \rightarrow \operatorname{Aut}(W)$ is an isomorphism of group schemes.

The automorphism group scheme of the general $W(m ; \underline{n})$ has also been determined by [Waterhouse 1991] for $p>2$ and with small exceptions for $p=3$, and by [Skryabin 1995; 2001] for any $p$, with small exceptions in the cases $p=2$ and $p=3$. In particular, Theorem 3.1 holds for $p=3$ if $m \geq 2$ and for $p=2$ if $m \geq 3$. 
In this section we establish analogues of Theorem 3.1 for the simple algebras $S(m ; \underline{1})^{(1)}$ and $H(m ; \underline{1})^{(2)}$. We follow the approach of [Waterhouse 1971]. Suppose $\Phi: \mathbf{G} \rightarrow \mathbf{H}$ is a morphism of algebraic group schemes. Let $\mathbf{G}_{\mathrm{red}}$ and $\mathbf{H}_{\mathrm{red}}$ be the largest smooth subgroupschemes, which will be regarded as algebraic groups. In order for $\Phi$ to be an isomorphism, the following two conditions are necessary:
A) The restriction $\Phi: \mathbf{G}_{\text {red }} \rightarrow \mathbf{H}_{\text {red }}$ is a bijection.
B) The tangent map $\operatorname{Lie}(\Phi): \operatorname{Lie}(\mathbf{G}) \rightarrow \operatorname{Lie}(\mathbf{H})$ is a bijection.

However, unless $\mathbf{G}$ is known to be smooth (that is, $\mathbf{G}=\mathbf{G}_{\text {red }}$ ), these two conditions are not sufficient for $\Phi$ to be an isomorphism. In general, one has to show that the associated map of distribution algebras $\widetilde{\Phi}: \mathfrak{G} \rightarrow \mathfrak{H}$ is surjective. (The two conditions above imply that $\widetilde{\Phi}: \mathfrak{G} \rightarrow \mathfrak{H}$ is injective.)

Recall that the distribution algebra $\mathfrak{G}$ of an algebraic group scheme $\mathbf{G}$ is a connected cocommutative Hopf algebra (see, for example, [Montgomery 1993] for background on Hopf algebras), with the space of primitive elements $\operatorname{Prim}(\mathfrak{G})=$ $\operatorname{Lie}(\mathbf{G})$ of finite dimension. Hence $\operatorname{Lie}(\mathbf{G})$ has a descending chain of restricted Lie subalgebras - see, for example, [Sweedler 1967; Dieudonné 1973, II, Section 3, Number 2]:

$$
\operatorname{Lie}(\mathbf{G})=\operatorname{Lie}^{0}(\mathbf{G}) \supset \operatorname{Lie}^{1}(\mathbf{G}) \supset \operatorname{Lie}^{2}(\mathbf{G}) \supset \cdots,
$$

defined by $\operatorname{Lie}^{k}(\mathbf{G}):=\operatorname{Lie}^{k}(\mathfrak{G})=\mathfrak{V}^{k}(\mathfrak{G}) \cap \operatorname{Prim}(\mathfrak{G})$, where $\mathfrak{V}: \mathfrak{G} \rightarrow \mathfrak{G}$ is the Verschiebung operator - see, for example, [Sweedler 1967, Theorem 1; Dieudonné 1973, II, Section 2, Number 7]. The intersection of this chain is $\operatorname{Lie}\left(\mathbf{G}_{\mathrm{red}}\right)$, which can be identified with the tangent algebra of the algebraic group $\mathbf{G}_{\text {red }}$.

Recall that a sequence of elements $1={ }^{0} h,{ }^{1} h, \ldots,{ }^{n} h$ in a connected cocommutative Hopf algebra $\mathfrak{G}$ is called a sequence of divided powers (lying over ${ }^{1} h$ ) if $\Delta\left({ }^{j} h\right)=\sum_{i=0}^{j} h \otimes{ }^{j-i} h$ for all $j=1, \ldots, n$. Thus ${ }^{1} h \in \operatorname{Prim}(\mathfrak{G})$ and $\varepsilon\left({ }^{j} h\right)=0$ for all $j=1, \ldots, n$. It is easy to see that $\mathfrak{V}^{k}\left(p^{k} h\right)={ }^{1} h$ for any $p^{k} \leq n$. Hence, if there exists a sequence of divided powers of length $p^{k}$ lying over $h \in \operatorname{Prim}(\mathfrak{G})$, then $h \in \operatorname{Lie}^{k}(\mathfrak{G})$. The converse is also true [Sweedler 1967, Theorem 2].

Now we come back to the problem of proving that a morphism $\Phi: \mathbf{G} \rightarrow \mathbf{H}$ of algebraic group schemes is an isomorphism. Assuming that $\Phi$ satisfies conditions A) and B), we need to show that the Hopf subalgebra $\widetilde{\Phi}(\mathfrak{G}) \subset \mathfrak{H}$ in fact equals $\mathfrak{H}$. Regarding $\mathfrak{H}$ as a Hopf subalgebra in the distribution algebra of GL(V) for a suitable space $V$, we can apply [Dieudonné 1973, II, Section 3, Number 2, Corollary 1] to conclude that $\widetilde{\Phi}(\mathfrak{G})=\mathfrak{H}$ if and only if

C) $\operatorname{Lie}(\Phi)$ maps $\operatorname{Lie}^{k}(\mathbf{G})$ onto $\operatorname{Lie}^{k}(\mathbf{H})$ for all $k$.

Here we are interested in the case $\mathbf{G}=\operatorname{Aut}(\mathcal{O})$, where $\mathbb{O}=\mathbb{O}(m ; \underline{1})$, and its subgroupschemes $\operatorname{Aut}_{S}(\mathbf{O}):=\operatorname{Stab}_{\mathbf{G}}\left(\left\langle\omega_{S}\right\rangle\right)$ and $\operatorname{Aut}_{H}(\mathbf{O}):=\operatorname{Stab}_{\mathbf{G}}\left(\left\langle\omega_{H}\right\rangle\right)$. We 
have

$$
\begin{aligned}
\operatorname{Aut}(\mathcal{O})_{\mathrm{red}} & =\operatorname{Aut}(\mathcal{O}), & \operatorname{Lie}(\operatorname{Aut}(\mathcal{O})) & =\operatorname{Der}(\mathcal{O})=W, \\
\operatorname{Aut}_{S}(\mathcal{O})_{\mathrm{red}} & =\operatorname{Stab}_{\operatorname{Aut}(\mathcal{O})}\left(\left\langle\omega_{S}\right\rangle\right), & \operatorname{Lie}\left(\operatorname{Aut}_{S}(\mathcal{O})\right) & =\operatorname{Stab}_{W}\left(\left\langle\omega_{S}\right\rangle\right)=: C S, \\
\operatorname{Aut}_{H}(\mathcal{O})_{\mathrm{red}} & =\operatorname{Stab}_{\operatorname{Aut}(\mathcal{O})}\left(\left\langle\omega_{H}\right\rangle\right), & \operatorname{Lie}\left(\operatorname{Aut}_{H}(\mathcal{O})\right) & =\operatorname{Stab}_{W}\left(\left\langle\omega_{H}\right\rangle\right)=: C H .
\end{aligned}
$$

Denote $\operatorname{Stab}_{\text {Aut(C) }}\left(\left\langle\omega_{S}\right\rangle\right)$ by $\operatorname{Aut}_{S}(\mathcal{O})$ and $\operatorname{Stab}_{\operatorname{Aut}(\mathcal{O})}\left(\left\langle\omega_{H}\right\rangle\right)$ by $\operatorname{Aut}_{H}(\mathcal{O})$ for brevity.

Assume $p>3$. It is known that the morphism Ad : $\operatorname{Aut}(O) \rightarrow \operatorname{Aut}(W)$ as well as its restrictions $\operatorname{Aut}_{S}(\mathcal{O}) \rightarrow \operatorname{Aut}\left(S^{(1)}\right)$ for $m \geq 3$ and $\operatorname{Aut}_{H}(\mathcal{O}) \rightarrow \operatorname{Aut}\left(H^{(2)}\right)$ for $m=2 r$ induce bijections $\operatorname{Aut}(\mathbb{O}) \rightarrow \operatorname{Aut}(W), \operatorname{Aut}_{S}(\mathbb{O}) \rightarrow \operatorname{Aut}\left(S^{(1)}\right)$ and $\operatorname{Aut}_{H}(\mathbb{O}) \rightarrow$ $\operatorname{Aut}\left(H^{(2)}\right)$ - see, for example, [Strade 2004, Theorem 7.3.2]. Also, the tangent map ad: $W \rightarrow \operatorname{Der}(W)$ and its restrictions $C S \rightarrow \operatorname{Der}\left(S^{(1)}\right)$ and $C H \rightarrow \operatorname{Der}\left(H^{(2)}\right)$ are bijective - see, for example, [Strade 2004, Theorem 7.1.2]. So conditions A) and B) are satisfied for the morphisms $\operatorname{Aut}(\mathcal{O}) \rightarrow \operatorname{Aut}(W), \operatorname{Aut}_{S}(\mathbb{O}) \rightarrow \operatorname{Aut}\left(S^{(1)}\right)$ and $\operatorname{Aut}_{H}(\mathbf{O}) \rightarrow \operatorname{Aut}\left(H^{(2)}\right)$.

By [Allen and Sweedler 1969, Lemma 3.5, 2], we know that $\operatorname{Lie}^{k}(\operatorname{Aut}(\mathbb{O}))=$ $\operatorname{Stab}_{W}(\mathfrak{M})=W_{(0)}$ for all $k>0$. Hence, for $k>0$, we have

$$
\begin{gathered}
\operatorname{Lie}^{k}\left(\operatorname{Aut}_{S}(0)\right) \subset \operatorname{Lie}^{k}(\operatorname{Aut}(O)) \cap C S=C S_{(0)}, \\
\operatorname{Lie}^{k}\left(\operatorname{Aut}_{H}(0)\right) \subset \operatorname{Lie}^{k}(\operatorname{Aut}(O)) \cap C H=C H_{(0)} .
\end{gathered}
$$

On the other hand, $\operatorname{Lie}\left(\operatorname{Aut}_{S}\left(O^{\circ}\right)\right)=C S_{(0)}$ and $\operatorname{Lie}\left(\operatorname{Aut}_{H}(\mathcal{O})\right)=C H_{(0)}$. It follows that, in fact,

$$
\operatorname{Lie}^{k}\left(\operatorname{Aut}_{S}(0)\right)=C S_{(0)} \quad \text { and } \quad \operatorname{Lie}^{k}\left(\operatorname{Aut}_{H}(0)\right)=C H_{(0)} \quad \text { for all } k>0 .
$$

By [Allen and Sweedler 1969, Lemma 3.5, 4], we have $\operatorname{Lie}^{k}(\operatorname{Aut}(W))=\operatorname{ad}\left(W_{(0)}\right)$ for all $k>0$, so condition C) is satisfied for the morphism $\operatorname{Aut}(\mathcal{O}) \rightarrow \operatorname{Aut}(W)$. This is how Theorem 3.1 is proved in [Waterhouse 1971]. We are now ready to prove our analogues for $S$ and $H$.

Theorem 3.2. Let $\mathbb{O}=\mathcal{O}(m ; \underline{1})$ for $m \geq 3$ and $S^{(1)}=S(m ; \underline{1})^{(1)}$. Let

$$
\operatorname{Aut}_{S}(\mathbb{O})=\operatorname{Stab}_{\text {Aut }(\mathcal{O})}\left(\left\langle\omega_{S}\right\rangle\right) .
$$

Assume $p>3$. Then the morphism $\operatorname{Ad}: \operatorname{Aut}_{S}(\mathbb{0}) \rightarrow \operatorname{Aut}\left(S^{(1)}\right)$ is an isomorphism of group schemes.

Proof. By the above discussion, we have to prove that condition C) is satisfied for $\operatorname{Ad}: \operatorname{Aut}_{S}(\mathcal{O}) \rightarrow \operatorname{Aut}\left(S^{(1)}\right)$. Let $\mathfrak{G}$ be the distribution algebra of $\operatorname{Aut}\left(S^{(1)}\right)$. In view of (1), it suffices to show that $\operatorname{Lie}^{1}(\mathfrak{G}) \subset \operatorname{ad}\left(C S_{(0)}\right)$. In other words, we have to verify, for any $D \in C S$, that if $D \notin C S_{(0)}$, then ad $D \notin \operatorname{Lie}^{1}(\mathfrak{G})$. We can write $D=\lambda_{1} \partial_{1}+\cdots+\lambda_{m} \partial_{m}+D_{0}$ where $D_{0} \in C S_{(0)}$ and the scalars $\lambda_{1}, \ldots, \lambda_{m}$ are not all zero. Now, $D_{0} \in \operatorname{Lie}^{1}\left(\operatorname{Aut}_{S}(\mathbb{O})\right)$ implies ad $D_{0} \in \operatorname{Lie}^{1}(\mathfrak{G})$, so it suffices to prove 
that $\operatorname{ad}\left(\lambda_{1} \partial_{1}+\cdots+\lambda_{m} \partial_{m}\right) \notin \operatorname{Lie}^{1}(\mathfrak{G})$. Applying an automorphism of $\mathbb{O}$ induced by a suitable linear transformation on the space $\operatorname{Span}\left\{\partial_{1}, \ldots, \partial_{m}\right\}$, we may assume without loss of generality that $D=\partial_{1}$.

By way of contradiction, assume that ad $\partial_{1} \in \operatorname{Lie}^{1}(\mathfrak{G})$. Then there exists a sequence of divided powers $1={ }^{0} h,{ }^{1} h, \ldots,{ }^{p} h$ in $\mathfrak{G}$ such that ${ }^{1} h=\operatorname{ad} \partial_{1}$.

As pointed out in the proof of [Allen and Sweedler 1969, Lemma 3.5, 4], by [Sweedler 1967, Lemma 7], we may assume without loss of generality that ${ }^{k} h=(1 / k !)\left({ }^{1} h\right)^{k}$ for $k=0, \ldots, p-1$. The distribution algebra $\mathfrak{G}$ acts canonically on $S^{(1)}$, so we have a homomorphism $\eta: \mathfrak{G} \rightarrow \operatorname{End}\left(S^{(1)}\right)$. The restriction of $\eta$ to $\operatorname{Prim}(\mathfrak{G})=\operatorname{Der}\left(S^{(1)}\right)$ is the identity map. Let ${ }^{k} \delta=\eta\left({ }^{k} h\right)$ for $k=0, \ldots, p$. Then

$$
{ }^{k} \delta=\frac{1}{k !}\left(\operatorname{ad} \partial_{1}\right)^{k} \quad \text { for } k=0, \ldots, p-1,
$$

and, since $S^{(1)}$ is a $\mathfrak{G}$-module algebra,

$$
{ }^{k} \delta([X, Y])=\sum_{j=0}^{k}\left[{ }^{j} \delta(X),{ }^{k-j} \delta(Y)\right] \text { for all } k=0, \ldots, p \text { and } X, Y \in S^{(1)} .
$$

The action of $\mathfrak{G}$ on $S^{(1)}$ extends canonically to the universal enveloping algebra $U\left(S^{(1)}\right)$ and, since the $p$-map of $S^{(1)}$ is uniquely determined, the $\mathfrak{G}$-action passes on to the restricted enveloping algebra $u\left(S^{(1)}\right)$. By abuse of notation, we will use ${ }^{k} \delta$ to denote the action of ${ }^{k} h$ on $u\left(S^{(1)}\right)$ as well as on $S^{(1)}$. Note for future reference that

(4) ${ }^{k} \delta(X Y)=\sum_{j=0}^{k}\left({ }^{j} \delta\right)(X)\left({ }^{k-j} \delta\right)(Y) \quad$ for all $k=0, \ldots, p$ and $X, Y \in u\left(S^{(1)}\right)$.

If we replace ${ }^{p} \delta$ by ${ }^{p} \delta+\xi$, where $\xi$ is any derivation of $S^{(1)}$, then Equations (3) and (4) will still hold (with the same ${ }^{0} \delta, \ldots,{ }^{p-1} \delta$ ). We will use this observation to simplify the operator ${ }^{p} \delta$.

Let $z_{i}=1+x_{i}, i=1, \ldots, m$. For each multi-index $\alpha \in \mathbb{Z}^{(m ; 1)}$, set

$$
z^{\alpha}=z_{1}^{\alpha_{1}} \cdots z_{m}^{\alpha_{m}}
$$

Since $z_{i}^{p}=1$ for all $i$, we may regard the components of $\alpha$ as elements of the cyclic group $\mathbb{Z}_{p}$ when dealing with $z^{\alpha}$. It is this property that will make the basis $\left\{z^{\alpha}\right\}$ of $\mathcal{O}=\mathcal{O}(m ; \underline{1})$ more convenient for us than the standard basis $\left\{x^{\alpha}\right\}$.

Recall [Strade 2004, Section 4.2] that $S^{(1)}=S(m ; \underline{1})^{(1)}$ is spanned by the elements of the form

$$
D_{i, j}(f):=\partial_{j}(f) \partial_{i}-\partial_{i}(f) \partial_{j},
$$


where $f \in \mathcal{O}$ and $1 \leq i<j \leq m$. Hence, $S^{(1)}$ is spanned by the elements

$$
D_{i, j}\left(z^{\alpha}\right)=\alpha_{j} z^{\alpha-\varepsilon_{j}} \partial_{i}-\alpha_{i} z^{\alpha-\varepsilon_{i}} \partial_{j} .
$$

For the calculations we are about to carry out, we will need the following:

Lemma 3.3. For any $1 \leq i<j \leq m$ and $\alpha, \beta \in \mathbb{Z}^{(m ; 1)}$, the commutator

$$
\left[D_{1,2}\left(z^{\alpha}\right), D_{i, j}\left(z^{\beta}\right)\right]
$$

is given by the following expressions:

$$
\begin{array}{cl}
-\left(\alpha_{1} \beta_{2}-\alpha_{2} \beta_{1}\right) D_{i, j}\left(z^{\alpha+\beta-\varepsilon_{1}-\varepsilon_{2}}\right) & \text { if } 2<i<j, \\
-\left(\alpha_{1}\left(\beta_{2}-1\right)-\alpha_{2} \beta_{1}\right) D_{2, j}\left(z^{\alpha+\beta-\varepsilon_{1}-\varepsilon_{2}}\right) & \text { if } 2=i<j, \\
-\alpha_{1} \beta_{j} D_{1,2}\left(z^{\alpha+\beta-\varepsilon_{1}-\varepsilon_{j}}\right)+\alpha_{2} \beta_{1} D_{1, j}\left(z^{\alpha+\beta-\varepsilon_{1}-\varepsilon_{2}}\right) & \\
-\alpha_{1} \beta_{1} D_{2, j}\left(z^{\alpha+\beta-2 \varepsilon_{1}}\right) & \text { if } i=1, j>2, \\
-\left(\alpha_{1} \beta_{2}-\alpha_{2} \beta_{1}\right) D_{1,2}\left(z^{\alpha+\beta-\varepsilon_{1}-\varepsilon_{2}}\right) & \text { if } i=1, j=2 .
\end{array}
$$

Proof. The verification of expressions (5)-(8) is straightforward. Here we will verify (7), which is somewhat special, and leave the rest to the reader. We have, for $j>2$,

$$
\begin{array}{r}
{\left[D_{1,2}\left(z^{\alpha}\right), D_{1, j}\left(z^{\beta}\right)\right]=\left[\alpha_{2} z^{\alpha-\varepsilon_{2}} \partial_{1}-\alpha_{1} z^{\alpha-\varepsilon_{1}} \partial_{2}, \beta_{j} z^{\beta-\varepsilon_{j}} \partial_{1}-\beta_{1} z^{\beta-\varepsilon_{1}} \partial_{j}\right]} \\
=\quad \alpha_{2} \beta_{j} \beta_{1} z^{\alpha+\beta-\varepsilon_{1}-\varepsilon_{2}-\varepsilon_{j}} \partial_{1}-\alpha_{2} \beta_{j} \alpha_{1} z^{\alpha+\beta-\varepsilon_{1}-\varepsilon_{2}-\varepsilon_{j}} \partial_{1} \\
\quad+\alpha_{2} \beta_{1} \alpha_{j} z^{\alpha+\beta-\varepsilon_{1}-\varepsilon_{2}-\varepsilon_{j}} \partial_{1}-\alpha_{2} \beta_{1}\left(\beta_{1}-1\right) z^{\alpha+\beta-2 \varepsilon_{1}-\varepsilon_{2}} \partial_{j} \\
\quad+\alpha_{1} \beta_{j}\left(\alpha_{1}-1\right) z^{\alpha+\beta-2 \varepsilon_{1}-\varepsilon_{j}} \partial_{2}-\alpha_{1} \beta_{j} \beta_{2} z^{\alpha+\beta-\varepsilon_{1}-\varepsilon_{2}-\varepsilon_{j}} \partial_{1} \\
\quad+\alpha_{1} \beta_{1} \beta_{2} z^{\alpha+\beta-2 \varepsilon_{1}-\varepsilon_{2}} \partial_{j}-\alpha_{1} \beta_{1} \alpha_{j} z^{\alpha+\beta-2 \varepsilon_{1}-\varepsilon_{j}} \partial_{2} \\
=\left(\alpha_{2} \beta_{j} \beta_{1}-\alpha_{2} \beta_{j} \alpha_{1}+\alpha_{2} \beta_{1} \alpha_{j}-\alpha_{1} \beta_{j} \beta_{2}\right) z^{\alpha+\beta-\varepsilon_{1}-\varepsilon_{2}-\varepsilon_{j}} \partial_{1} \\
\quad+\alpha_{1}\left(\beta_{j}\left(\alpha_{1}-1\right)-\beta_{1} \alpha_{j}\right) z^{\alpha+\beta-2 \varepsilon_{1}-\varepsilon_{j}} \partial_{2} \\
+\beta_{1}\left(-\alpha_{2}\left(\beta_{1}-1\right)+\alpha_{1} \beta_{2}\right) z^{\alpha+\beta-2 \varepsilon_{1}-\varepsilon_{2}} \partial_{j} .
\end{array}
$$

Comparing the above with

$$
\begin{aligned}
D_{1,2}\left(z^{\alpha+\beta-\varepsilon_{1}-\varepsilon_{j}}\right) & =\left(\alpha_{2}+\beta_{2}\right) z^{\alpha+\beta-\varepsilon_{1}-\varepsilon_{2}-\varepsilon_{j}} \partial_{1}-\left(\alpha_{1}+\beta_{1}-1\right) z^{\alpha+\beta-2 \varepsilon_{1}-\varepsilon_{j}} \partial_{2}, \\
D_{1, j}\left(z^{\alpha+\beta-\varepsilon_{1}-\varepsilon_{2}}\right) & =\left(\alpha_{j}+\beta_{j}\right) z^{\alpha+\beta-\varepsilon_{1}-\varepsilon_{2}-\varepsilon_{j}} \partial_{1}-\left(\alpha_{1}+\beta_{1}-1\right) z^{\alpha+\beta-2 \varepsilon_{1}-\varepsilon_{2}} \partial_{j}, \\
D_{2, j}\left(z^{\alpha+\beta-2 \varepsilon_{1}}\right) & =\left(\alpha_{j}+\beta_{j}\right) z^{\alpha+\beta-2 \varepsilon_{1}-\varepsilon_{j}} \partial_{2}-\left(\alpha_{2}+\beta_{2}\right) z^{\alpha+\beta-2 \varepsilon_{1}-\varepsilon_{2}} \partial_{j},
\end{aligned}
$$

one readily sees that (7) holds.

Another useful fact is the following:

$$
\left[\partial_{\ell}, D_{i, j}(f)\right]=D_{i, j}\left(\partial_{\ell}(f)\right) \text { for all } i, j, \ell=1, \ldots, m \text { and } f \in \mathcal{O} \text {. }
$$

The remaining part of the proof of Theorem 3.2 will be divided into four steps. 
Step 1: Without loss of generality, we may assume

$$
{ }^{p} \delta\left(D_{1,2}\left(z_{1} z_{2}\right)\right)=0 \text {. }
$$

Substituting $\alpha=\varepsilon_{1}+\varepsilon_{2}$ into expressions (5)-(8), we see that each nonzero element $D_{i, j}\left(z^{\beta}\right)$ is an eigenvector for the operator ad $D_{1,2}\left(z_{1} z_{2}\right)$, with eigenvalue $\lambda(i, j, \beta)=\beta_{1}-\beta_{2}-1, \beta_{1}-\beta_{2}$ or $\beta_{1}-\beta_{2}+1$, depending on $i, j$. (For the case $i=1$ and $j>2$, one has to combine the first and the third terms in expression (7), which gives $-\left(\beta_{2}+1\right) D_{1, j}\left(z^{\beta}\right)$.) Since $\lambda(i, j, \beta)$ is in the field $G F_{p}$, we have $D_{1,2}\left(z_{1} z_{2}\right)=D_{1,2}\left(z_{1} z_{2}\right)^{p}$. Applying the operator ${ }^{p} \delta$ to both sides and using (4), we obtain

$$
{ }^{p} \delta\left(D_{1,2}\left(z_{1} z_{2}\right)\right)=\sum_{i_{1}+\cdots+i_{p}=p}\left({ }^{i_{1}} \delta\right)\left(D_{1,2}\left(z_{1} z_{2}\right)\right) \cdots\left({ }^{i_{p}} \delta\right)\left(D_{1,2}\left(z_{1} z_{2}\right)\right) .
$$

Taking into account (2) and (9), we see that ${ }^{k} \delta\left(D_{1,2}\left(z_{1} z_{2}\right)\right)=0$ for $1<k<p$. Hence,

$$
\begin{aligned}
{ }^{p} \delta\left(D_{1,2}\left(z_{1} z_{2}\right)\right)=\left({ }^{1} \delta\right. & \left.\left(D_{1,2}\left(z_{1} z_{2}\right)\right)\right)^{p} \\
& +\sum_{k=0}^{p-1} D_{1,2}\left(z_{1} z_{2}\right)^{k}\left({ }^{p} \delta\right)\left(D_{1,2}\left(z_{1} z_{2}\right)\right) D_{1,2}\left(z_{1} z_{2}\right)^{p-k-1} .
\end{aligned}
$$

Since ${ }^{1} \delta\left(D_{1,2}\left(z_{1} z_{2}\right)\right)=\left[\partial_{1}, D_{1,2}\left(z_{1} z_{2}\right)\right]=D_{1,2}\left(z_{2}\right)=\partial_{1}$ and $\partial_{1}^{p}=0$, the first term on the right-hand side of (11) vanishes. The second term can be rewritten using the identity

$$
\sum_{k=0}^{p-1} X^{k} Y X^{p-k-1}=(\operatorname{ad} X)^{p-1}(Y),
$$

where $X=D_{1,2}\left(z_{1} z_{2}\right)$ and $Y={ }^{p} \delta(X)$. Thus, (11) yields

$$
Y=\left(\operatorname{ad} D_{1,2}\left(z_{1} z_{2}\right)\right)^{p-1}(Y) .
$$

It follows that $Y$ can be written as a linear combination of those $D_{i, j}\left(z^{\beta}\right)$ for which the eigenvalue $\lambda(i, j, \beta)$ is nonzero:

$$
{ }^{p} \delta\left(D_{1,2}\left(z_{1} z_{2}\right)\right)=\sum_{i, j, \beta: \lambda(i, j, \beta) \neq 0} \sigma_{\beta}^{i, j} D_{i, j}\left(z^{\beta}\right) .
$$

Replacing ${ }^{p} \delta$ with

$$
{ }^{p} \delta+\sum_{i, j, \beta} \frac{\sigma_{\beta}^{i, j}}{\lambda(i, j, \beta)} \text { ad } D_{i, j}\left(z^{\beta}\right)
$$

completes Step 1. 
Step 2: Without loss of generality, we may assume, in addition to (10), that

$$
{ }^{p} \delta\left(\partial_{1}\right)=\sum_{i, j, \beta: \beta_{1}=p-1} \tau_{\beta}^{i, j} D_{i, j}\left(z^{\beta}\right)
$$

for some scalars $\tau_{\beta}^{i, j}$.

By (9), we have $\left[\partial_{1}, D_{1,2}\left(z_{1} z_{2}\right)\right]=\partial_{1}$. Applying the operator ${ }^{p} \delta$ to both sides and using (3), (2) and (10), we obtain

$$
{ }^{p} \delta\left(\partial_{1}\right)=\sum_{k=0}^{p}\left[{ }^{k} \delta\left(\partial_{1}\right),{ }^{p-k} \delta\left(D_{1,2}\left(z_{1} z_{2}\right)\right)\right]=\left[{ }^{p} \delta\left(\partial_{1}\right), D_{1,2}\left(z_{1} z_{2}\right)\right] .
$$

Hence $\left[D_{1,2}\left(z_{1} z_{2}\right), Y\right]=-Y$ where $Y={ }^{p} \delta\left(\partial_{1}\right)$. It follows that $Y$ can be written as a linear combination of those $D_{i, j}\left(z^{\beta}\right)$ for which the eigenvalue $\lambda(i, j, \beta)$ is -1 :

$$
{ }^{p} \delta\left(\partial_{1}\right)=\sum_{i, j, \beta: \lambda(i, j, \beta)=-1} \tau_{\beta}^{i, j} D_{i, j}\left(z^{\beta}\right) .
$$

Now replace ${ }^{p} \delta$ with

$$
{ }^{p} \delta+\sum_{i, j, \beta: \beta_{1} \neq p-1} \frac{\tau_{\beta}^{i, j}}{\beta_{1}+1} \operatorname{ad} D_{i, j}\left(z^{\beta+\varepsilon_{1}}\right) .
$$

Using (9) gives (12) for the new ${ }^{p} \delta$, because all terms with $\beta_{1} \neq p-1$ in the righthand side of (13) will cancel out. It remains to check that we still have (10) for the new ${ }^{p} \delta$. In other words, we have to check that $\left[D_{1,2}\left(z_{1} z_{2}\right), D_{i, j}\left(z^{\beta+\varepsilon_{1}}\right)\right]=0$ for all $i, j, \beta$ with $\tau_{\beta}^{i, j} \neq 0$. But this is clear, because $\lambda\left(i, j, \beta+\varepsilon_{1}\right)=\lambda(i, j, \beta)+1$ and thus $\lambda\left(i, j, \beta+\varepsilon_{1}\right)=0$ for all $i, j, \beta$ that occur in the right-hand side of (13). Step 2 is complete.

Step 3: For any element $X=f_{1} \partial_{1}+\cdots+f_{m} \partial_{m} \in W$, define

$$
\operatorname{pr}_{1}(X):=f_{1} \in \mathcal{O} \text {. }
$$

Assume (10) and (12). Then, $\operatorname{pr}_{1}\left({ }^{p} \delta\left(D_{1,2}\left(z_{1}^{k} z_{2}\right)\right)\right)$ for any $k=1, \ldots, p-1$ is a linear combination of $z^{\gamma}$ with $0 \leq \gamma_{1}<k$.

We proceed by induction on $k$. The basis for $k=1$ follows from (10). Now suppose the claim holds for some $k \geq 1$. By (9), we have

$$
\left[\partial_{1}, D_{1,2}\left(z_{1}^{k+1} z_{2}\right)\right]=(k+1) D_{1,2}\left(z_{1}^{k} z_{2}\right) .
$$

Applying the operator ${ }^{p} \delta$ to both sides, we obtain

$$
{ }^{p} \delta\left(\left[\partial_{1}, D_{1,2}\left(z_{1}^{k+1} z_{2}\right)\right]\right)=(k+1)\left({ }^{p} \delta\right)\left(D_{1,2}\left(z_{1}^{k} z_{2}\right)\right) .
$$


Using (3),(2) and (12), the left-hand side of (14) becomes

$$
\left[\sum_{i, j, \beta: \beta_{1}=p-1} \tau_{\beta}^{i, j} D_{i, j}\left(z^{\beta}\right), D_{1,2}\left(z_{1}^{k+1} z_{2}\right)\right]+\left[\partial_{1},{ }^{p} \delta\left(D_{1,2}\left(z_{1}^{k+1} z_{2}\right)\right)\right] .
$$

Setting $Y={ }^{p} \delta\left(D_{1,2}\left(z_{1}^{k+1} z_{2}\right)\right)$, we can rewrite (14) as

$$
\left[\partial_{1}, Y\right]=(k+1)\left({ }^{p} \delta\right)\left(D_{1,2}\left(z_{1}^{k} z_{2}\right)\right)+\sum_{i, j, \beta: \beta_{1}=p-1} \tau_{\beta}^{i, j}\left[D_{1,2}\left(z_{1}^{k+1} z_{2}\right), D_{i, j}\left(z^{\beta}\right)\right] .
$$

Our goal is to show that monomials $z^{\gamma}$ with $\gamma_{1} \geq k+1$ do not occur in $f_{1}:=\operatorname{pr}_{1}(Y)$. Since $\operatorname{pr}_{1}\left[\partial_{1}, Y\right]=\partial_{1} f_{1}$, it suffices to show that elements $z^{\gamma} \partial_{1}$ with $\gamma_{1} \geq k$ do not occur in the right-hand side of (15), when it is regarded as an element of $W$. The induction hypothesis tells us that such elements do not occur in the first term of the right-hand side of (15). We will prove the same for the second term.

In the case $2<i<j$, we have by (5) that

$$
\left[D_{1,2}\left(z_{1}^{k+1} z_{2}\right), D_{i, j}\left(z^{\beta}\right)\right]=\left(\beta_{1}-\beta_{2}(k+1)\right) D_{i, j}\left(z^{\beta+k \varepsilon_{1}}\right) .
$$

Hence, no elements $z^{\gamma} \partial_{1}$ occur here.

In the case $2=i<j$, we have by (6) that

$$
\left[D_{1,2}\left(z_{1}^{k+1} z_{2}\right), D_{i, j}\left(z^{\beta}\right)\right]=\left(\beta_{1}-\left(\beta_{2}-1\right)(k+1)\right) D_{2, j}\left(z^{\beta+k \varepsilon_{1}}\right) .
$$

Again, no elements $z^{\gamma} \partial_{1}$ occur.

In the case $i=1$ and $2<j$, we can write [ $\left.D_{1,2}\left(z_{1}^{k+1} z_{2}\right), D_{i, j}\left(z^{\beta}\right)\right]$, using (7) and $\beta_{1}=p-1$, as

$$
-(k+1) \beta_{j} D_{1,2}\left(z^{\beta+k \varepsilon_{1}+\varepsilon_{2}-\varepsilon_{j}}\right)-D_{1, j}\left(z^{\beta+k \varepsilon_{1}}\right)+(k+1) D_{2, j}\left(z^{\beta+(k-1) \varepsilon_{1}+\varepsilon_{2}}\right) .
$$

Elements $z^{\gamma} \partial_{1}$ occur only in the first two summands, and we have $\gamma=\beta+k \varepsilon_{1}-\varepsilon_{j}$ in either case. Therefore, $\gamma_{1}=\beta_{1}+k=k-1 \bmod p($ recall that we may take the exponents of $z$ modulo $p$ ).

In the case $i=1, j=2$, we have by $\beta_{1}=p-1$ and (8) that

$$
\left[D_{1,2}\left(z_{1}^{k+1} z_{2}\right), D_{1,2}\left(z^{\beta}\right)\right]=-\left((k+1) \beta_{2}+1\right) D_{1,2}\left(z^{\beta+k \varepsilon_{1}}\right) .
$$

Hence, elements $z^{\gamma} \partial_{1}$ occur with $\gamma=\beta+k \varepsilon_{1}-\varepsilon_{2}$. Once again, $\gamma_{1}=k-1 \bmod p$. The inductive proof of Step 3 is complete.

Step 4: We can finally obtain a contradiction. By (8), we have

$$
\left[D_{1,2}\left(z_{1}^{p-1} z_{2}\right), D_{1,2}\left(z_{1}^{2} z_{2}\right)\right]=3 D_{1,2}\left(z_{2}\right)=3 \partial_{1} .
$$


Applying ${ }^{p} \delta$ and taking into account (3), (2) and (12), we obtain

$$
\begin{aligned}
& 3 \sum_{i, j, \beta: \beta_{1}=p-1} \tau_{\beta}^{i, j} D_{i, j}\left(z^{\beta}\right) \\
& =\left[{ }^{p} \delta\left(D_{1,2}\left(z_{1}^{p-1} z_{2}\right)\right), D_{1,2}\left(z_{1}^{2} z_{2}\right)\right]+\left[D_{1,2}\left(z_{1}^{p-1} z_{2}\right),{ }^{p} \delta\left(D_{1,2}\left(z_{1}^{2} z_{2}\right)\right)\right] \\
& +\left[D_{1,2}\left(\frac{1}{(p-1) !} \partial_{1}^{p-1}\left(z_{1}^{p-1} z_{2}\right)\right), D_{1,2}\left(\frac{1}{1 !} \partial_{1}\left(z_{1}^{2} z_{2}\right)\right)\right] \\
& \quad+\left[D_{1,2}\left(\frac{1}{(p-2) !} \partial_{1}^{p-2}\left(z_{1}^{p-1} z_{2}\right)\right), D_{1,2}\left(\frac{1}{2 !} \partial_{1}^{2}\left(z_{1}^{2} z_{2}\right)\right)\right] .
\end{aligned}
$$

One readily verifies that the sum of the third and fourth terms in the right-hand side of (16) is $3 \partial_{1}$. Consider the first and the second terms. Denote

$$
X:={ }^{p} \delta\left(D_{1,2}\left(z_{1}^{p-1} z_{2}\right)\right) \quad \text { and } \quad Y:={ }^{p} \delta\left(D_{1,2}\left(z_{1}^{2} z_{2}\right)\right) .
$$

By Step 3, we know $\operatorname{pr}_{1}(X)$ is a linear combination of $z^{\gamma}$ with $0 \leq \gamma_{1} \leq p-2$ and $\operatorname{pr}_{1}(Y)$ is a linear combination of $z^{\gamma}$ with $0 \leq \gamma_{1} \leq 1$.

Since $D_{1,2}\left(z_{1}^{2} z_{2}\right)=z_{1}^{2} \partial_{1}-2 z_{1} z_{2} \partial_{2}$, we see that the coefficient of $\partial_{1}$ depends only on $z_{1}$ and hence all terms with $\partial_{1}$ in the commutator $\left[X, D_{1,2}\left(z_{1}^{2} z_{2}\right)\right]$ come from terms with $\partial_{1}$ in $X$. In other words,

$$
\operatorname{pr}_{1}\left[X, D_{1,2}\left(z_{1}^{2} z_{2}\right)\right]=\operatorname{pr}_{1}\left[\operatorname{pr}_{1}(X) \partial_{1}, D_{1,2}\left(z_{1}^{2} z_{2}\right)\right] \text {. }
$$

Since

$$
\left[z^{\gamma} \partial_{1}, D_{1,2}\left(z_{1}^{2} z_{2}\right)\right]=\left(2-\gamma_{1}+2 \gamma_{2}\right) z^{\gamma+\varepsilon_{1}} \partial_{1}-2 z^{\gamma+\varepsilon_{2}} \partial_{2},
$$

we conclude that $\operatorname{pr}_{1}\left[X, D_{1,2}\left(z_{1}^{2} z_{2}\right)\right]$ is a linear combination of monomials $z^{\gamma+\varepsilon_{1}}$ with $0 \leq \gamma_{1} \leq p-2$. Therefore, elements $z^{\alpha} \partial_{1}$ with $\alpha_{1}=0$ do not occur in the first term in the right-hand side of (16).

Since $D_{1,2}\left(z_{1}^{p-1} z_{2}\right)=z_{1}^{p-1} \partial_{1}+z_{1}^{p-2} z_{2} \partial_{2}$, we also see that

$$
\operatorname{pr}_{1}\left[D_{1,2}\left(z_{1}^{p-1} z_{2}\right), Y\right]=\operatorname{pr}_{1}\left[D_{1,2}\left(z_{1}^{p-1} z_{2}\right), \operatorname{pr}_{1}(Y) \partial_{1}\right] \text {. }
$$

Then

$$
\left[D_{1,2}\left(z_{1}^{p-1} z_{2}\right), z^{\gamma} \partial_{1}\right]=\left(1+\gamma_{1}+\gamma_{2}\right) z^{\gamma+(p-2) \varepsilon_{1}} \partial_{1}+2 z^{\gamma+(p-3) \varepsilon_{1}+\varepsilon_{2}} \partial_{2},
$$

implies that $\operatorname{pr}_{1}\left[D_{1,2}\left(z_{1}^{p-1} z_{2}\right), Y\right]$ is a linear combination of monomials $z^{\gamma+(p-2) \varepsilon_{1}}$ with $0 \leq \gamma_{1} \leq 1$. Therefore, elements $z^{\alpha} \partial_{1}$ with $\alpha_{1}=0$ do not occur in the second term in the right-hand side of (16).

Finally, all elements $z^{\alpha} \partial_{1}$ that occur in the left-hand side of (16) have $\alpha_{1}=p-1$. Summarizing our analysis, we obtain

$$
3 \partial_{1}=0,
$$

which is a contradiction, since $p>3$. The proof of Theorem 3.2 is complete. 
Corollary 3.4. Under the assumptions of Theorem 3.2,

$$
\operatorname{Lie}^{k}\left(\operatorname{Aut}\left(S^{(1)}\right)\right)=\operatorname{ad}\left(C S_{(0)}\right) \quad \text { for all } k>0 .
$$

Theorem 3.5. Let $\mathcal{O}=\mathcal{O}(m ; \underline{1})$ for $m=2 r$ and $H^{(2)}=H(m ; \underline{1})^{(2)}$. Let

$$
\operatorname{Aut}_{H}(\mathcal{O})=\operatorname{Stab}_{\mathbf{A u t}(\mathcal{O})}\left(\left\langle\omega_{H}\right\rangle\right) .
$$

Assume $p>3$. Then the morphism $\mathrm{Ad}: \operatorname{Aut}_{H}(\mathbf{O}) \rightarrow \operatorname{Aut}\left(H^{(2)}\right)$ is an isomorphism of group schemes.

Proof. Denote by $\mathfrak{G}$ the distribution algebra of $\operatorname{Aut}\left(H^{(2)}\right)$. As in the proof of Theorem 3.2, we must show that $\operatorname{Lie}^{1}(\mathfrak{G}) \subset \operatorname{ad}\left(C H_{(0)}\right)$ - that is, for any $D \in C H$, if $D \notin C H_{(0)}$, then ad $D \notin \operatorname{Lie}^{1}(\mathfrak{G})$. Again, we can write $D=\lambda_{1} \partial_{1}+\cdots+\lambda_{m} \partial_{m}+D_{0}$, where $D_{0} \in C H_{(0)}$ and the scalars $\lambda_{1}, \ldots, \lambda_{m}$ are not all zero. Since $D_{0}$ is in $\operatorname{Lie}^{1}\left(\operatorname{Aut}_{H}(\mathcal{O})\right)$, we have that ad $D_{0}$ is in $\operatorname{Lie}^{1}(\mathfrak{G})$, so it suffices to prove that $\operatorname{ad}\left(\lambda_{1} \partial_{1}+\cdots+\lambda_{m} \partial_{m}\right) \notin \operatorname{Lie}^{1}(\mathfrak{G})$. Applying an automorphism of $\mathbb{O}$ induced by a suitable symplectic transformation on the space $\operatorname{Span}\left\{\partial_{1}, \ldots, \partial_{m}\right\}$, we may assume without loss of generality that $D=\partial_{1}$.

By way of contradiction, assume that ad $\partial_{1} \in \operatorname{Lie}^{1}(\mathfrak{G})$. Then there exists a sequence of divided powers $1={ }^{0} h,{ }^{1} h, \ldots,{ }^{p} h$ in $\mathfrak{G}$ such that ${ }^{1} h=\operatorname{ad} \partial_{1}$. We may assume without loss of generality that ${ }^{k} h=(1 / k !)\left({ }^{1} h\right)^{k}$ for $k=0, \ldots, p-1$.

The distribution algebra $\mathfrak{G}$ acts canonically on $H^{(2)}$, so there is a homomorphism $\eta: \mathfrak{G} \rightarrow \operatorname{End}\left(H^{(2)}\right)$. The restriction of $\eta$ to $\operatorname{Prim}(\mathfrak{G})=\operatorname{Der}\left(H^{(2)}\right)$ is the identity map. Let ${ }^{k} \delta=\eta\left({ }^{k} h\right)$ for $k=0, \ldots, p$. Then

$$
{ }^{k} \delta=\frac{1}{k !}\left(\operatorname{ad} \partial_{1}\right)^{k} \quad \text { for } k=0, \ldots, p-1,
$$

and, since $H^{(2)}$ is a $\mathfrak{G}$-module algebra,

$$
{ }^{k} \delta([X, Y])=\sum_{j=0}^{k}\left[{ }^{j} \delta(X),{ }^{k-j} \delta(Y)\right] \quad \text { for all } k=0, \ldots, p \text { and } X, Y \in H^{(2)} .
$$

For the extended action of $\mathfrak{G}$ on the restricted enveloping algebra $u\left(H^{(2)}\right)$, we have

$$
{ }^{k} \delta(X Y)=\sum_{j=0}^{k}\left({ }^{j} \delta\right)(X)\left({ }^{k-j} \delta\right)(Y) \quad \text { for all } k=0, \ldots, p \text { and } X, Y \in u\left(H^{(2)}\right) .
$$

We may replace ${ }^{p} \delta$ by ${ }^{p} \delta+\xi$, where $\xi$ is any derivation of $H^{(2)}$, without affecting Equations (18) and (19). We will use this observation to simplify the operator ${ }^{p} \delta$.

As in [Strade 2004, Section 4.2], define

$$
\sigma(i):=\left\{\begin{array}{ll}
1 & \text { if } i=1, \ldots, r, \\
-1 & \text { if } i=r+1, \ldots, 2 r,
\end{array} \quad \text { and } \quad i^{\prime}:=i+\sigma(i) r .\right.
$$


Also define a map $D_{H}: \mathcal{O} \rightarrow H$ by

$$
D_{H}(f):=\sum_{i=1}^{2 r} \sigma(i) \partial_{i}(f) \partial_{i^{\prime}} .
$$

Note that the kernel of $D_{H}$ is $\mathbb{F} 1$.

Let $z_{i}=1+x_{i}, i=1, \ldots, 2 r$. For each multi-index $\alpha \in \mathbb{Z}^{(2 r ; 1)}$, set

$$
z^{\alpha}=z_{1}^{\alpha_{1}} \cdots z_{2 r}^{\alpha_{2 r}}
$$

We may regard the components of $\alpha$ as elements of the cyclic group $\mathbb{Z}_{p}$ when dealing with $z^{\alpha}$. For the calculations we are about to carry out, we need the formula

$$
\left[D_{H}\left(z^{\alpha}\right), D_{H}\left(z^{\beta}\right)\right]=D_{H}\left(D_{H}\left(z^{\alpha}\right)\left(z^{\beta}\right)\right) \quad \text { for all } \alpha, \beta \in \mathbb{Z}^{(2 r ; 1)} \text {. }
$$

In particular,

$$
\left[\partial_{\ell}, D_{H}\left(z^{\beta}\right)\right]=D_{H}\left(\partial_{\ell}\left(z^{\beta}\right)\right) \quad \text { for all } \ell=1, \ldots, 2 r \text { and } \beta \in \mathbb{Z}^{(2 r ; \underline{1})} .
$$

Let $\tau=(p-1, \ldots, p-1) \in \mathbb{Z}^{(2 r ; 1)}$. By [Strade 2004, Section 4.2],

$$
\left\{D_{H}\left(z^{\alpha}\right) \mid 0<\alpha<\tau\right\}
$$

is a basis of $H^{(2)}$.

The remaining part of the proof of Theorem 3.5 will be divided into four steps, which are similar to the steps in the proof of Theorem 3.2.

Step 1: Without loss of generality, we may assume

$$
{ }^{p} \delta\left(D_{H}\left(z_{1} z_{r+1}\right)\right)=0 .
$$

Substituting $\alpha=\varepsilon_{1}+\varepsilon_{2}$ into (20), we see that $D_{H}\left(z^{\beta}\right)$ is an eigenvector for the operator ad $D_{H}\left(z_{1} z_{r+1}\right)$, with eigenvalue $\beta_{r+1}-\beta_{1}$. Since $\beta_{r+1}-\beta_{1}$ is in the field $G F_{p}$, we have $D_{H}\left(z_{1} z_{r+1}\right)=D_{H}\left(z_{1} z_{r+1}\right)^{p}$. Applying the operator ${ }^{p} \delta$ to both sides and using (19), we obtain

$$
p_{\delta}\left(D_{H}\left(z_{1} z_{r+1}\right)\right)=\sum_{i_{1}+\cdots+i_{p}=p}\left({ }^{i_{1}} \delta\right)\left(D_{H}\left(z_{1} z_{r+1}\right)\right) \cdots\left({ }^{i_{p}} \delta\right)\left(D_{H}\left(z_{1} z_{r+1}\right)\right) .
$$

Taking into account (17) and (21), we see that ${ }^{k} \delta\left(D_{H}\left(z_{1} z_{r+1}\right)\right)=0$ for $1<k<p$. Hence,

$$
\begin{aligned}
p^{p} \delta\left(D_{H}\left(z_{1} z_{r+1}\right)\right) & =\left({ }^{1} \delta\left(D_{H}\left(z_{1} z_{r+1}\right)\right)\right)^{p} \\
& +\sum_{k=0}^{p-1} D_{H}\left(z_{1} z_{r+1}\right)^{k}\left({ }^{p} \delta\right)\left(D_{H}\left(z_{1} z_{r+1}\right)\right) D_{H}\left(z_{1} z_{r+1}\right)^{p-k-1} .
\end{aligned}
$$


Since ${ }^{1} \delta\left(D_{H}\left(z_{1} z_{r+1}\right)\right)=\left[\partial_{1}, D_{H}\left(z_{1} z_{r+1}\right)\right]=D_{H}\left(z_{r+1}\right)=-\partial_{1}$ and $\partial_{1}^{p}=0$, the first term on the right-hand side of (23) vanishes. The second term can be rewritten using the identity

$$
\sum_{k=0}^{p-1} X^{k} Y X^{p-k-1}=(\operatorname{ad} X)^{p-1}(Y),
$$

where $X=D_{H}\left(z_{1} z_{r+1}\right)$ and $Y={ }^{p} \delta(X)$. Thus, (23) yields

$$
Y=\left(\operatorname{ad} D_{H}\left(z_{1} z_{r+1}\right)\right)^{p-1}(Y) .
$$

It follows that $Y$ is a linear combination of those $D_{H}\left(z^{\beta}\right)$ for which the eigenvalue $\beta_{r+1}-\beta_{1}$ is nonzero:

Replacing ${ }^{p} \delta$ with

$$
{ }^{p} \delta\left(D_{H}\left(z_{1} z_{r+1}\right)\right)=\sum_{\beta: \beta_{r+1} \neq \beta_{1}} \sigma_{\beta} D_{H}\left(z^{\beta}\right) .
$$

$$
{ }^{p} \delta+\sum_{\beta} \frac{\sigma_{\beta}}{\beta_{r+1}-\beta_{1}} \text { ad } D_{H}\left(z^{\beta}\right)
$$

completes Step 1.

Step 2: Without loss of generality, we may assume that, in addition to (22),

$$
{ }^{p} \delta\left(\partial_{1}\right)=\sum_{\beta: \beta_{1}=p-1} \tau_{\beta} D_{H}\left(z^{\beta}\right)
$$

for some scalars $\tau_{\beta}$.

By (21), we have $\left[\partial_{1}, D_{H}\left(z_{1} z_{r+1}\right)\right]=-\partial_{1}$. Applying the operator ${ }^{p} \delta$ to both sides and using (18), (17) and (22), we obtain

$$
-{ }^{p} \delta\left(\partial_{1}\right)=\sum_{k=0}^{p}\left[{ }^{k} \delta\left(\partial_{1}\right),{ }^{p-k} \delta\left(D_{H}\left(z_{1} z_{r+1}\right)\right)\right]=\left[{ }^{p} \delta\left(\partial_{1}\right), D_{H}\left(z_{1} z_{r+1}\right)\right] .
$$

Hence $\left[D_{H}\left(z_{1} z_{r+1}\right), Y\right]=Y$, where $Y={ }^{p} \delta\left(\partial_{1}\right)$. It follows that $Y$ is a linear combination of those $D_{H}\left(z^{\beta}\right)$ for which the eigenvalue $\beta_{r+1}-\beta_{1}$ is 1 :

$$
{ }^{p} \delta\left(\partial_{1}\right)=\sum_{\beta: \beta_{r+1}-\beta_{1}=1} \tau_{\beta} D_{H}\left(z^{\beta}\right) .
$$

Now replace ${ }^{p} \delta$ with

$$
{ }^{p} \delta+\sum_{\beta: \beta_{1} \neq p-1} \frac{\tau_{\beta}}{\beta_{1}+1} \text { ad } D_{H}\left(z^{\beta+\varepsilon_{1}}\right) .
$$

Using (21) gives (24) for the new ${ }^{p} \delta$, because all terms with $\beta_{1} \neq p-1$ in the righthand side of (25) will cancel out. It remains to check that we still have (22) for the new ${ }^{p} \delta$. In other words, we have to check that $\left[D_{H}\left(z_{1} z_{r+1}\right), D_{H}\left(z^{\beta+\varepsilon_{1}}\right)\right]=0$ 
for all $\beta$ with $\tau_{\beta} \neq 0$. But this is clear, because $\beta_{r+1}-\left(\beta_{1}+1\right)=0$ for all $\beta$ that occur in the right-hand side of (25). Step 2 is complete.

Step 3: Assume (22) and (24). Then, ${ }^{p} \delta\left(D_{H}\left(z_{1}^{k} z_{r+1}\right)\right)$ for any $k=1, \ldots, p-1$ is a linear combination of $D_{H}\left(z^{\gamma}\right)$ with $0 \leq \gamma_{1}<k$.

We proceed by induction on $k$. The basis for $k=1$ follows from (22). Now suppose the claim holds for some $k \geq 1$. By (21), we have

$$
\left[\partial_{1}, D_{H}\left(z_{1}^{k+1} z_{r+1}\right)\right]=(k+1) D_{H}\left(z_{1}^{k} z_{r+1}\right) .
$$

Applying the operator ${ }^{p} \delta$ to both sides and taking into account (18) and (17) gives

$$
\begin{array}{r}
{\left[{ }^{p} \delta\left(\partial_{1}\right), D_{H}\left(z_{1}^{k+1} z_{r+1}\right)\right]+\left[\partial_{1},{ }^{p} \delta\left(D_{H}\left(z_{1}^{k+1} z_{r+1}\right)\right)\right]} \\
=(k+1)\left({ }^{p} \delta\right)\left(D_{H}\left(z_{1}^{k} z_{r+1}\right)\right) .
\end{array}
$$

Writing

$$
{ }^{p} \delta\left(D_{H}\left(z_{1}^{k+1} z_{r+1}\right)\right)=\sum_{0<\gamma<\tau} \sigma_{\gamma} D_{H}\left(z^{\gamma}\right)
$$

and considering (24), (20) and (21), we can rewrite the left-hand side of (26) as

$$
\begin{aligned}
& \sum_{\beta: \beta_{1}=p-1} \tau_{\beta}\left[D_{H}\left(z^{\beta}\right), D_{H}\left(z_{1}^{k+1} z_{r+1}\right)\right]+\sum_{\gamma} \sigma_{\gamma}\left[\partial_{1}, D_{H}\left(z^{\gamma}\right)\right] \\
= & -\sum_{\beta: \beta_{1}=p-1} \tau_{\beta} D_{H}\left(\left((k+1) z_{1}^{k} z_{r+1} \partial_{r+1}-z_{1}^{k+1} \partial_{1}\right)\left(z^{\beta}\right)\right)+\sum_{\gamma} \sigma_{\gamma} D_{H}\left(\partial_{1}\left(z^{\gamma}\right)\right) \\
& =-\sum_{\beta: \beta_{1}=p-1} \tau_{\beta} D_{H}\left((k+1) \beta_{r+1} z^{\beta+k \varepsilon_{1}}-\beta_{1} z^{\beta+k \varepsilon_{1}}\right)+\sum_{\gamma} \sigma_{\gamma} \gamma_{1} D_{H}\left(z^{\gamma-\varepsilon_{1}}\right) \\
& =-\sum_{\beta: \beta_{1}=p-1} \tau_{\beta}\left((k+1) \beta_{r+1}+1\right) D_{H}\left(z^{\beta+k \varepsilon_{1}}\right)+\sum_{\gamma} \sigma_{\gamma} \gamma_{1} D_{H}\left(z^{\gamma-\varepsilon_{1}}\right) .
\end{aligned}
$$

Setting $\tau_{\beta}^{\prime}:=\tau_{\beta}\left((k+1) \beta_{r+1}+1\right)$, we can now rewrite (26) as

$$
\sum_{\gamma} \sigma_{\gamma} \gamma_{1} D_{H}\left(z^{\gamma-\varepsilon_{1}}\right)=(k+1)\left({ }^{p} \delta\right)\left(D_{H}\left(z_{1}^{k} z_{r+1}\right)\right)+\sum_{\beta: \beta_{1}=p-1} \tau_{\beta}^{\prime} D_{H}\left(z^{\beta+k \varepsilon_{1}}\right) .
$$

Since the induction hypothesis applies to the first term in the right-hand side of (28), all elements $D_{H}\left(z^{\alpha}\right)$ that occur in the right-hand side have $0 \leq \alpha_{1} \leq k-1$. Comparing this with the left-hand side, we conclude that for any $\gamma$ with $\sigma_{\gamma} \neq 0$, either $\gamma_{1}=0$ or $0 \leq \gamma_{1}-1 \leq k-1$. Hence the elements $D_{H}\left(z^{\gamma}\right)$ that occur in the right-hand side of (27) have $0 \leq \gamma_{1} \leq k$. The inductive proof of Step 3 is complete.

Step 4: We can finally obtain a contradiction. By (20), we have

$$
\left[D_{H}\left(z_{1}^{2} z_{r+1}\right), D_{H}\left(z_{1}^{p-1} z_{r+1}\right)\right]=3 D_{H}\left(z_{r+1}\right)=-3 \partial_{1} .
$$


Applying ${ }^{p} \delta$ and taking into account (18) and (17), we obtain

$$
\begin{array}{r}
-3\left({ }^{p} \delta\right)\left(\partial_{1}\right) \\
=\left[{ }^{p} \delta\left(D_{H}\left(z_{1}^{2} z_{r+1}\right)\right), D_{H}\left(z_{1}^{p-1} z_{r+1}\right)\right]+\left[D_{H}\left(z_{1}^{2} z_{r+1}\right),{ }^{p} \delta\left(D_{H}\left(z_{1}^{p-1} z_{r+1}\right)\right)\right] \\
+\left[D_{H}\left(\frac{1}{1 !} \partial_{1}\left(z_{1}^{2} z_{r+1}\right)\right), D_{H}\left(\frac{1}{(p-1) !} \partial_{1}^{p-1}\left(z_{1}^{p-1}\right) z_{r+1}\right)\right] \\
+\left[D_{H}\left(\frac{1}{2 !} \partial_{1}^{2}\left(z_{1}^{2} z_{r+1}\right)\right), D_{H}\left(\frac{1}{(p-2) !} \partial_{1}^{p-2}\left(z_{1}^{p-1}\right) z_{r+1}\right)\right] \\
=\left[{ }^{p} \delta\left(D_{H}\left(z_{1}^{2} z_{r+1}\right)\right), D_{H}\left(z_{1}^{p-1} z_{r+1}\right)\right]+\left[D_{H}\left(z_{1}^{2} z_{r+1}\right),{ }^{p} \delta\left(D_{H}\left(z_{1}^{p-1} z_{r+1}\right)\right)\right] \\
\quad+3\left[D_{H}\left(z_{1} z_{r+1}\right), D_{H}\left(z_{r+1}\right)\right] \\
=\left[{ }^{p} \delta\left(D_{H}\left(z_{1}^{2} z_{r+1}\right)\right), D_{H}\left(z_{1}^{p-1} z_{r+1}\right)\right]+\left[D_{H}\left(z_{1}^{2} z_{r+1}\right),{ }^{p} \delta\left(D_{H}\left(z_{1}^{p-1} z_{r+1}\right)\right)\right]-3 \partial_{1} .
\end{array}
$$

So we have

$$
\begin{aligned}
3 \partial_{1}=\left[{ }^{p} \delta\left(D_{H}\left(z_{1}^{2} z_{r+1}\right)\right)\right. & \left., D_{H}\left(z_{1}^{p-1} z_{r+1}\right)\right] \\
+ & {\left[D_{H}\left(z_{1}^{2} z_{r+1}\right),{ }^{p} \delta\left(D_{H}\left(z_{1}^{p-1} z_{r+1}\right)\right)\right]+3\left({ }^{p} \delta\right)\left(\partial_{1}\right) . }
\end{aligned}
$$

Step 3 gives that

$$
\begin{aligned}
p_{\delta}\left(D_{H}\left(z_{1}^{2} z_{r+1}\right)\right) & =\sum_{\alpha: 0 \leq \alpha_{1} \leq 1} \sigma_{\alpha}^{(2)} D_{H}\left(z^{\alpha}\right), \\
p_{\delta}\left(D_{H}\left(z_{1}^{p-1} z_{r+1}\right)\right) & =\sum_{\alpha: 0 \leq \alpha_{1} \leq p-2} \sigma_{\alpha}^{(p-1)} D_{H}\left(z^{\alpha}\right) .
\end{aligned}
$$

Hence, (20) implies

$$
\begin{aligned}
{ }^{p} \delta\left(D_{H}\left(z_{1}^{2} z_{r+1}\right)\right), & \left.D_{H}\left(z_{1}^{p-1} z_{r+1}\right)\right] \\
& =\sum_{\alpha: 0 \leq \alpha_{1} \leq 1} \sigma_{\alpha}^{(2)}\left[D_{H}\left(z^{\alpha}\right), D_{H}\left(z_{1}^{p-1} z_{r+1}\right)\right] \\
& =-\sum_{\alpha: 0 \leq \alpha_{1} \leq 1} \sigma_{\alpha}^{(2)} D_{H}\left(\left(-z_{1}^{p-2} z_{r+1} \partial_{r+1}-z_{1}^{p-1} \partial_{1}\right)\left(z^{\alpha}\right)\right) \\
& =\sum_{\alpha: 0 \leq \alpha_{1} \leq 1} \sigma_{\alpha}^{(2)}\left(\alpha_{r+1}+\alpha_{1}\right) D_{H}\left(z^{\alpha-2 \varepsilon_{1}}\right),
\end{aligned}
$$

and, similarly,

$$
\begin{aligned}
{\left[D_{H}\left(z_{1}^{2} z_{r+1}\right),{ }^{p} \delta\left(D_{H}\left(z_{1}^{p-1} z_{r+1}\right)\right)\right] } & =\sum_{\alpha: 0 \leq \alpha_{1} \leq p-2} \sigma_{\alpha}^{(p-1)}\left[D_{H}\left(z_{1}^{2} z_{r+1}\right), D_{H}\left(z^{\alpha}\right)\right] \\
& =\sum_{\alpha: 0 \leq \alpha_{1} \leq p-2} \sigma_{\alpha}^{(p-1)} D_{H}\left(\left(2 z_{1} z_{r+1} \partial_{r+1}-z_{1}^{2} \partial_{1}\right)\left(z^{\alpha}\right)\right) \\
& =\sum_{\alpha: 0 \leq \alpha_{1} \leq p-2} \sigma_{\alpha}^{(p-1)}\left(2 \alpha_{r+1}-\alpha_{1}\right) D_{H}\left(z^{\alpha+\varepsilon_{1}}\right) .
\end{aligned}
$$


Using these calculations and (24), we can rewrite (29) as

$$
\begin{aligned}
3 \partial_{1}= & \sum_{\alpha: 0 \leq \alpha_{1} \leq 1} \sigma_{\alpha}^{(2)}\left(\alpha_{r+1}+\alpha_{1}\right) D_{H}\left(z^{\alpha-2 \varepsilon_{1}}\right) \\
& +\sum_{\alpha: 0 \leq \alpha_{1} \leq p-2} \sigma_{\alpha}^{(p-1)}\left(2 \alpha_{r+1}-\alpha_{1}\right) D_{H}\left(z^{\alpha+\varepsilon_{1}}\right)+3 \sum_{\beta: \beta_{1}=p-1} \tau_{\beta} D_{H}\left(z^{\beta}\right) .
\end{aligned}
$$

This equation is impossible, because $p>3$ and none of the sums in the right-hand side involves $D_{H}\left(z_{r+1}\right)$. The proof of Theorem 3.5 is complete.

Corollary 3.6. Under the assumptions of Theorem 3.5,

$$
\operatorname{Lie}^{k}\left(\operatorname{Aut}\left(H^{(2)}\right)\right)=\operatorname{ad}\left(C H_{(0)}\right) \quad \text { for all } k>0 .
$$

\section{Group gradings}

Let $G$ be an abelian group. In this section we will give a classification of $G$ gradings on the algebra $\mathrm{O}=\mathcal{O}(m ; \underline{1})$ and on the simple Lie algebras $W(m ; \underline{1})$, $S(m ; \underline{1})^{(1)}$ for $m \geq 3$ and $S(2 ; \underline{1})^{(2)}=H(2 ; \underline{1})^{(2)}$.

Given a $G$-grading $\Gamma_{\mathscr{O}}: \mathcal{O}=\bigoplus_{g \in G} \mathscr{O}_{g}$, we obtain an induced grading on End(O). It is easy to see that $W=\operatorname{Der}(0)$ is a graded subspace, so it inherits a $G$-grading, which will be denoted by $\Gamma_{W}: W=\bigoplus_{g \in G} W_{g}$. The spaces $\Omega^{k}$ also receive $G$-gradings in a natural way, and one can verify that the maps $d: \Omega^{k} \rightarrow \Omega^{k+1}$ respect the $G$-gradings. (The canonical $\mathbb{Z}$-gradings of $W$ and $\Omega^{k}$ are induced by the canonical $\mathbb{Z}$-grading of $O$ in this manner.) However, $S=S(m ; \underline{1})$, respectively $H=H(m ; \underline{1})$, is not in general a graded subspace of $W$. It is certainly a graded subspace if we assume that $\omega_{S}$, respectively $\omega_{H}$, is a homogeneous element with respect to the $G$-grading on $\Omega^{m}$, respectively $\Omega^{2}$.

Definition 4.1. We will say that a $G$-grading $\Gamma_{\mathscr{O}}: \mathcal{O}=\bigoplus_{g \in G} \mathscr{O}_{g}$ is $S$-admissible of degree $g_{0} \in G$ if the form $\omega_{S}$ is a homogeneous element of degree $g_{0}$, and similarly define $H$-admissible.

If $\Gamma_{\odot}$ is $S$-admissible, then we denote the induced $G$-gradings on $S$ and its derived algebra(s) by $\Gamma_{S}$, and similarly use $\Gamma_{H}$ when $\Gamma_{\odot}$ is $H$-admissible.

We now recall the connection between group gradings on an algebra and certain subgroupschemes of its automorphism group scheme. Let $U$ be an algebra. For any group $G$, a $G$-grading on $U$ is equivalent to a structure of an $\mathbb{E} G$-comodule algebra (see, for example, [Montgomery 1993]). Assuming $U$ finite-dimensional and $G$ finitely generated abelian, we can regard this comodule structure as a morphism of algebraic group schemes $G^{D} \rightarrow \operatorname{Aut}(U)$ where $G^{D}$ is the Cartier dual of $G$, that is, the group scheme represented by the commutative Hopf algebra $\mathbb{F} G$. Two $G$ gradings are isomorphic if and only if the corresponding morphisms $G^{D} \rightarrow \operatorname{Aut}(U)$ are conjugate by an automorphism of $U$. 
If char $\mathbb{F}=0$, then $G^{D}=\widehat{G}$, the algebraic group of multiplicative characters of $G$, and $\operatorname{Aut}(U)=\operatorname{Aut}(U)$, the algebraic group of automorphisms. The image of $\widehat{G}$ in $\operatorname{Aut}(U)$ is a quasitorus, that is, a diagonalizable algebraic group. The $G$-grading on $U$ is, of course, the eigenspace decomposition of $U$ with respect to this quasitorus. Hence, group gradings on $U$ correspond to quasitori in $\operatorname{Aut}(U)$.

We are interested in the case char $\mathbb{F}=p>0$. Then we can write $G=G_{p^{\prime}} \times G_{p}$, where $G_{p^{\prime}}$ has no $p$-torsion and $G_{p}$ is a $p$-group. So $G^{D}=\widehat{G_{p^{\prime}}} \times G_{p}^{D}$, where $\widehat{G_{p^{\prime}}} \widehat{\text { is }}$ smooth and $G_{p}^{D}$ is finite and connected. The algebraic group $\widehat{G_{p^{\prime}}}$ (which equals $\widehat{G}$ ) is a quasitorus, and it acts by automorphisms of $U$ as follows:

$$
\chi * X=\chi(g) X \quad \text { for all } X \in U_{g} \text { and } g \in G .
$$

If $G_{p}$ is an elementary $p$-group, then the distribution algebra of $G_{p}^{D}$ is the restricted enveloping algebra $u(T)$ where $T$ is the group of additive characters of $G_{p}$, regarded as an abelian restricted Lie algebra. If $\left\{a_{1}, \ldots, a_{s}\right\}$ is a basis of $G_{p}$ (as a vector space over the field $G F_{p}$ ), then the dual basis $\left\{t_{1}, \ldots, t_{s}\right\}$ of $T$ has the property $\left(t_{i}\right)^{p}=t_{i}$ for all $i$. Therefore, $T$ is a torus in the sense of restricted Lie algebras. It acts by derivations of $U$ as follows:

$$
t * X=t(g) X \quad \text { for all } X \in U_{g} \text { and } g \in G .
$$

If $G_{p}$ is not elementary, then the distribution algebra of $G_{p}^{D}$ is not generated by primitive elements and hence its action on $U$ does not reduce to derivations. Regardless of what the case may be, the image of $G^{D}$ in $\operatorname{Aut}(U)$ is a diagonalizable subgroupscheme. In some sense, the $G$-grading on $U$ is its eigenspace decomposition (see, for example, [Waterhouse 1979]).

Now, Theorems 3.1 (where, as was pointed out, one can include the cases $p=2$ and $p=3$ ), 3.2 and 3.5 give us the following corollary.

Corollary 4.2. Let $G$ be an abelian group. Let $L$ be one of the following simple Lie algebras: $W=W(m ; \underline{1})$ for $m \geq 3$ if $p=2$ and $m \geq 2$ if $p=3, S^{(1)}=S(m ; \underline{1})^{(1)}$ for $m \geq 3$ and $p>3$ or $H^{(2)}=H(m ; \underline{1})^{(2)}$ for $m=2 r$ and $p>3$. Then any $G$-grading on $L$ is induced by a $G$-grading on $\mathrm{O}=\mathrm{O}(m ; \underline{1})$. More precisely:

1) The correspondence $\Gamma_{\mathscr{O}} \mapsto \Gamma_{W}$ is a bijection between the G-gradings on 0 and the G-gradings on W. It induces a bijection between the isomorphism classes of these gradings.

2) The correspondence $\Gamma_{\mathscr{O}} \mapsto \Gamma_{S}$ is a bijection between the $S$-admissible $G$ gradings on $\mathcal{O}$ and the $G$-gradings on $S^{(1)}$. It induces a bijection between the isomorphism classes of G-gradings on $S^{(1)}$ and the $\mathrm{Aut}_{S}(\mathbb{O})$-orbits of the $S$-admissible $G$-gradings on $\mathrm{O}$.

3) The correspondence $\Gamma_{\odot} \mapsto \Gamma_{H}$ is a bijection between the $H$-admissible $G$ gradings on 0 and the G-gradings on $H^{(2)}$. It induces a bijection between 
the isomorphism classes of $G$-gradings on $H^{(2)}$ and the $\mathrm{Aut}_{H}(\mathrm{O})$-orbits of the $H$-admissible $G$-gradings on $\mathrm{O}$.

Proof. Let $\Gamma: L=\bigoplus_{g \in G} L_{g}$ be a $G$-grading. Replacing $G$ with the subgroup generated by the support, we may assume that $G$ is finitely generated. In addition, the corresponding morphism $G^{D} \rightarrow \operatorname{Aut}(L)$ is a closed imbedding.

Consider the case $L=W$. From the isomorphism Ad : $\operatorname{Aut}(0) \rightarrow \operatorname{Aut}(L)$, we obtain a closed imbedding $G^{D} \rightarrow \operatorname{Aut}(\mathcal{O})$, which corresponds to a $G$-grading $\Gamma_{\mathscr{O}}: \mathcal{O}=\bigoplus_{g \in G} \mathscr{O}_{g}$ (whose support also generates $G$, since otherwise we would not have a closed imbedding). The induced $G$-grading $\Gamma_{W}$ on $W$ is obtained by inducing the $\mathbb{F} G$-comodule structure from $\mathbb{O}$ to $\operatorname{End}(\mathcal{O})$ and then restricting to $L$, which agrees with how Ad: $\operatorname{Aut}(0) \rightarrow \operatorname{Aut}(L)$ is defined. Therefore, $\Gamma=\Gamma_{W}$.

In the case $L=S^{(1)}$, we obtain a closed imbedding $G^{D} \rightarrow \operatorname{Aut}_{S}(\mathbb{O})$, so the subspace $\left\langle\omega_{S}\right\rangle$ of $\Omega^{m}$ is $G^{D}$-invariant, that is, $\left\langle\omega_{S}\right\rangle$ is an $\mathbb{F} G$-subcomodule. Hence $\omega_{S}$ is a homogeneous element in the corresponding $G$-grading $\Gamma_{\mathscr{O}}: \mathcal{O}=\bigoplus_{g \in G} \mathbb{O}_{g}$.

The proof in the case $L=H^{(2)}$ is similar.

Remark 4.3. It follows from the proof that the supports of the gradings $\Gamma_{\mathscr{}}, \Gamma_{W}$, $\Gamma_{S}$ and $\Gamma_{H}$ generate the same subgroup in $G$.

We will now describe all possible $G$-gradings on $\mathcal{O}=\mathscr{O}(m ; \underline{1})$.

Proposition 4.4. Let $\mathbb{O}=\mathfrak{O}(m ; \underline{1})$ and let $\mathfrak{M}$ be its unique maximal ideal. Let $G$ be an abelian group and let $\mathrm{O}=\bigoplus_{g \in G} \mathcal{O}_{g}$ be a $G$-grading.

1) There exist elements $y_{1}, \ldots, y_{m}$ of $\mathfrak{M}$ and $0 \leq s \leq m$ such that the elements $1+y_{1}, \ldots, 1+y_{s}, y_{s+1}, \ldots, y_{m}$ are $G$-homogeneous and $\left\{y_{1}, \ldots, y_{m}\right\}$ is a basis of $\mathfrak{M}$ modulo $\mathfrak{M}^{2}$.

2) Let $P=\left\{g \in G \mid O_{g} \not \subset \mathfrak{M}\right\}$. Then $P$ is an elementary p-subgroup of $G$.

3) Let $\left\{b_{1}, \ldots, b_{s}\right\}$ be a basis of $P$. Then the elements $y_{1}, \ldots, y_{m}$ can be chosen in such a way that the degree of $1+y_{i}$ is $b_{i}$ for all $i=1, \ldots, s$.

Proof. 1) Pick a basis for 0 consisting of $G$-homogeneous elements and select a subset $\left\{f_{1}, \ldots, f_{m}\right\}$ of this basis that is linearly independent modulo $\mathbb{F} 1 \oplus \mathfrak{M}^{2}$. Order the elements $f_{i}$ so $f_{1}, \ldots, f_{s}$ have a nonzero constant term and $f_{s+1}, \ldots, f_{m}$ belong to $\mathfrak{M}$. Rescale $f_{1}, \ldots, f_{s}$ so that the constant term is 1 . Let $y_{i}=f_{i}-1$ for $i=1, \ldots, s$ and $y_{i}=f_{i}$ for $i=s+1, \ldots, m$. Then $y_{1}, \ldots, y_{m}$ is a basis of $\mathfrak{M}$ modulo $\mathfrak{M}^{2}$.

2) Clearly, $e \in P$. If $a, b \in P$, then there exist elements $u \in \mathbb{O}_{a}$ and $v \in \mathbb{O}_{b}$ that are not in $\mathfrak{M}$. Then the element $u v \in \mathbb{O}_{a b}$ is not in $\mathfrak{M}$, so $a b \in P$. Also, since $u^{p}$ is a nonzero scalar, we have $a^{p}=e$. Thus $P$ is an elementary $p$-subgroup. 
3) Any element of 0 can be uniquely written as a (truncated) polynomial in the variables $1+y_{1}, \ldots, 1+y_{s}, y_{s+1}, \ldots, y_{m}$. Hence, for any $g \in G$,

$$
\mathscr{O}_{g}=\operatorname{Span}\left\{\left(1+y_{1}\right)^{j_{1}} \cdots\left(1+y_{s}\right)^{j_{s}} y_{s+1}^{j_{s+1}} \cdots y_{m}^{j_{m}} \mid 0 \leq j_{i}<p, a_{1}^{j_{1}} \cdots a_{m}^{j_{m}}=g\right\}
$$

where $a_{1}, \ldots, a_{m} \in G$ are the degrees of $1+y_{1}, \ldots, 1+y_{s}, y_{s+1}, \ldots, y_{m}$, respectively. So $a_{1}, \ldots, a_{s}$ generate $P$. Suppose they do not form a basis of $P$ - say, $a_{s}=a_{1}^{\ell_{1}} \cdots a_{s-1}^{\ell_{s-1}}$. Set $\tilde{y}_{i}=y_{i}$ for $i \neq s$ and

$$
\tilde{y}_{s}:=1+y_{s}-\left(1+y_{1}\right)^{\ell_{1}} \cdots\left(1+y_{s-1}\right)^{\ell_{s-1}} .
$$

Then $1+\tilde{y}_{1}, \ldots, 1+\tilde{y}_{s-1}, \tilde{y}_{s}, \ldots, \tilde{y}_{m}$ are homogeneous of degrees $a_{1}, \ldots, a_{m}$, respectively. Also, $\tilde{y}_{s} \in \mathfrak{M}$ and

$$
\tilde{y}_{s}=y_{s}-\left(\ell_{1} y_{1}+\cdots+\ell_{s-1} y_{s-1}\right)\left(\bmod \mathfrak{M}^{2}\right),
$$

so $\tilde{y}_{1}, \ldots, \tilde{y}_{m}$ still form a basis of $\mathfrak{M}$ modulo $\mathfrak{M}^{2}$. We have decreased $s$ by 1 . Repeating this process as necessary, we may assume that $\left\{a_{1}, \ldots, a_{s}\right\}$ is a basis of $P$. Finally, if $\left\{b_{1}, \ldots, b_{s}\right\}$ is another basis of $P$, we can write $b_{j}=\prod_{i=1}^{s} a_{i}^{\ell_{i j}}$, where $\left(\ell_{i j}\right)$ is a nondegenerate matrix with entries in the field $G F_{p}$. Set

$$
\tilde{y}_{j}:=\prod_{i=1}^{s}\left(1+y_{i}\right)^{\ell_{i j}}-1 \quad \text { for } j=1, \ldots, s,
$$

and $\tilde{y}_{j}=y_{j}$ for $j=s+1, \ldots, m$. Then $\tilde{y}_{1}, \ldots, \tilde{y}_{m}$ form a basis of $\mathfrak{M}$ modulo $\mathfrak{M}^{2}$, and $1+\tilde{y}_{j}$ is homogeneous of degree $b_{j}, j=1, \ldots, s$.

Remark 4.5. Without loss of generality, assume that $G$ is generated by the support of the grading $\mathrm{O}=\bigoplus_{g \in G} \mathrm{O}_{g}$. Let $\mathbf{Q}$ be the image of $G^{D}$ under the corresponding closed imbedding $G^{D} \rightarrow \operatorname{Aut}(\mathcal{O})$. Let $\mathbf{H}=\operatorname{Stab}_{\operatorname{Aut}(\mathcal{O})}(\mathfrak{M})$. (In fact, $\mathbf{H}=\operatorname{Aut}\left(\mathcal{O}_{)}\right.$, regarded as the largest smooth subgroupscheme of $\operatorname{Aut}\left(\mathcal{O}^{\prime}\right)$.) Let $\mathbf{Q}_{0}=\mathbf{Q} \cap \mathbf{H}$. Then $P$ is the subgroup of $G$ corresponding to the Hopf ideal of $\mathbb{F} G$ defining the subgroupscheme $\mathbf{Q}_{0}$ of $\mathbf{Q}$.

Proof. Let $I_{0}$ be the Hopf ideal defining the subgroupscheme $\mathbf{Q}_{0}$ and let $G_{0}$ be the corresponding subgroup of $G$. Consider the coarsening $0=\bigoplus_{\bar{g} \in G / G_{0}} O_{\bar{g}}$ of the $G$ grading induced by the natural homomorphism $G \rightarrow G / G_{0}$, that is, $\bigcirc_{\bar{g}}=\bigoplus_{g \in \bar{g}} O_{g}$. This coarsening corresponds to the subgroupscheme $\mathbf{Q}_{0} \subset \mathbf{Q}$. Since $\mathbf{Q}_{0}$ stabilizes the subspace $\mathfrak{M} \subset \mathfrak{O}$, we have $\mathfrak{M}=\bigoplus_{\bar{g} \in G / G_{0}}\left(\mathscr{O}_{\bar{g}} \cap \mathfrak{M}\right)$. Hence $\mathfrak{O}_{\bar{g}} \subset \mathfrak{M}$ for $\bar{g} \neq \bar{e}$ and $\mathbb{O}_{\bar{e}}=\mathbb{F} 1 \oplus\left(\mathbb{O}_{\bar{e}} \cap \mathfrak{M}\right)$. Hence $\mathscr{O}_{g} \subset \mathfrak{M}$ for all $g \notin G_{0}$, which proves $P \subset G_{0}$. To prove that $P=G_{0}$, consider the Hopf ideal $I$ of $\mathbb{F} G$ corresponding to $P$. Then $I \subset I_{0}$. The subgroupscheme $\widetilde{\mathbf{Q}}$ of $\mathbf{Q}$ defined by $I$ acts trivially on each $\mathcal{O}_{g}$ with $g \in P$. It follows that $\widetilde{\mathbf{Q}}$ stabilizes $\mathfrak{M}$. Hence $\widetilde{\mathbf{Q}} \subset \mathbf{Q}_{0}$ and $I \supset I_{0}$. 
The description of the $G$-gradings on $\mathcal{O}(m ; \underline{1})$ resembles the description of the $G$-gradings on the matrix algebra $M_{n}(\mathbb{F})$ - see, for example, [Bahturin and Kochetov 2010; Elduque 2010]. Namely, Proposition 4.4 shows that the $G$-graded algebra $\mathbb{O}(m ; \underline{1})$ is isomorphic to the tensor product $\mathcal{O}(s ; \underline{1}) \otimes \mathbb{O}(m-s ; \underline{1})$ where the first factor has a division grading (in the sense that each homogeneous component is spanned by an invertible element) and the second factor has an elementary grading (in the sense that it is induced by a grading of the underlying vector space $\mathfrak{M} / \mathfrak{M}^{2}$ ). The isomorphism in question is, of course, the one defined by $y_{1} \mapsto x_{1} \otimes 1, \ldots, y_{s} \mapsto x_{s} \otimes 1$ and $y_{s+1} \mapsto 1 \otimes x_{1}, \ldots, y_{m} \mapsto 1 \otimes x_{m-s}$. The first factor, $\mathcal{O}(s ; \underline{1})$, is isomorphic to the group algebra $\mathbb{F} P$ as a $G$-graded algebra (where $\mathbb{F} P$ has the standard $P$-grading, which is regarded as a $G$-grading).

To state the classification of $G$-gradings on $\mathbb{O}$ up to isomorphism, we introduce some notation.

Definition 4.6. Let $P \subset G$ be an elementary $p$-subgroup of rank $s$ for $0 \leq s \leq m$. Let $t=m-s$ and let $\gamma=\left(g_{1}, \ldots, g_{t}\right) \in G^{t}$. Endow the algebra $\mathcal{O}=\mathscr{O}(m ; \underline{1})$ with a $G$-grading as follows. Select a basis $\left\{b_{1}, \ldots, b_{s}\right\}$ for $P$ and declare the degrees of $1+x_{1}, \ldots, 1+x_{s}$ to be $b_{1}, \ldots, b_{s}$, respectively. Declare the degrees of $x_{s+1}, \ldots, x_{m}$ to be $g_{1}, \ldots, g_{t}$, respectively. Denote the resulting $G$-grading on 0 by $\Gamma_{\mathscr{O}}\left(G, b_{1}, \ldots, b_{s}, g_{1}, \ldots, g_{t}\right)$. Since the gradings corresponding to different choices of basis for $P$ are isomorphic to each other, we also denote this grading (abusing notation) by $\Gamma_{\mathscr{O}}(G, P, \gamma)$.

Definition 4.7. Let $\gamma, \tilde{\gamma} \in G^{t}$. Write $\gamma \sim \tilde{\gamma}$ if there exists a permutation $\pi$ of the set $\{1, \ldots, t\}$ such that $\tilde{g}_{i} \equiv g_{\pi(i)}(\bmod P)$ for all $i=1, \ldots, t$.

Theorem 4.8. Let $\mathbb{F}$ be an algebraically closed field of characteristic $p>0$. Let $G$ be an abelian group. Let $\mathbb{O}=\bigoplus_{g \in G} \mathcal{O}_{g}$ be a grading on the algebra $\mathbb{O}=\mathbb{O}(m ; \underline{1})$ over $\mathbb{F}$. Then the grading is isomorphic to some $\Gamma_{\mathscr{O}}(G, P, \gamma)$ as in Definition 4.6. Two $G$-gradings $\Gamma_{\mathscr{O}}(G, P, \gamma)$ and $\Gamma_{\mathscr{O}}(G, \widetilde{P}, \tilde{\gamma})$ are isomorphic if and only if $P=\widetilde{P}$ and $\gamma \sim \tilde{\gamma}$ (see Definition 4.7).

Proof. Let $y_{1}, \ldots, y_{m}$ be as in Proposition 4.4. Let $g_{1}, \ldots, g_{t} \in G$ be the degrees of $y_{s+1}, \ldots, y_{m}$, respectively. Then the automorphism of 0 defined by

$$
y_{i} \mapsto x_{i} \quad \text { for } i=1, \ldots, m
$$

sends the grading $\mathbb{O}=\bigoplus_{g \in G} \mathcal{O}_{g}$ to $\Gamma_{\mathscr{O}}\left(G, b_{1}, \ldots, b_{s}, g_{1}, \ldots, g_{t}\right)$.

If $\tilde{g}_{i}=g_{\pi(i)}, i=1, \ldots, t$, for some permutation $\pi$, then the automorphism of 0 defined by

$$
x_{i} \mapsto x_{i} \text { for } i=1, \ldots, s \quad \text { and } \quad x_{s+i} \mapsto x_{s+\pi(i)} \text { for } i=1, \ldots, t,
$$

sends $\Gamma_{\mathscr{O}}(G, P, \tilde{\gamma})$ to $\Gamma_{\mathscr{O}}(G, P, \gamma)$. 
If $\tilde{g}_{i}=g_{i} b_{1}^{\ell_{i 1}} \cdots b_{s}^{\ell_{i s}}$, then the automorphism of $\mathcal{O}$ defined by

$$
x_{i} \mapsto x_{i} \text { for } i=1, \ldots, s \quad \text { and } \quad x_{s+i} \mapsto x_{s+i} \prod_{j=1}^{s}\left(1+x_{j}\right)^{\ell_{i j}} \text { for } i=1, \ldots, t,
$$

sends $\Gamma_{\mathscr{O}}(G, P, \tilde{\gamma})$ to $\Gamma_{\mathscr{O}}(G, P, \gamma)$.

Therefore, if $\gamma \sim \tilde{\gamma}$ as in Definition 4.7, then $\Gamma_{\mathscr{O}}(G, P, \tilde{\gamma})$ is isomorphic to $\Gamma_{\mathscr{O}}(G, P, \gamma)$.

It remains to show that the subgroup $P$ and the equivalence class of $\gamma$ are invariants of the $G$-graded algebra $\mathbb{O}=\bigoplus_{g \in G} \mathcal{O}_{g}$. This is obvious for $P$, since $P=\left\{g \in G \mid \mathcal{O}_{g} \not \subset \mathfrak{M}\right\}$. Let $\bar{G}=G / P$ and consider the coarsening of the $G$ grading, $\mathcal{O}=\bigoplus_{\bar{g} \in \bar{G}} \mathfrak{O}_{\bar{g}}$, induced by the natural homomorphism $G \rightarrow \bar{G}$. It follows from the definition of $P$ that $\mathfrak{M}$ is a $\bar{G}$-graded subspace of $\mathcal{O}$. Consequently, $\mathfrak{M}^{2}$ is also a $\bar{G}$-graded subspace, and the quotient $V:=\mathfrak{M} / \mathfrak{M}^{2}$ inherits a $\bar{G}$-grading:

$$
V=V_{\bar{a}_{1}} \oplus \cdots \oplus V_{\bar{a}_{\ell}}
$$

Define

$$
k_{i}= \begin{cases}\operatorname{dim} V_{\bar{a}_{i}} & \text { if } \bar{a}_{i} \neq \bar{e}, \\ \operatorname{dim} V_{\bar{a}_{i}}-s & \text { if } \bar{a}_{i}=\bar{e} .\end{cases}
$$

Clearly, $\bar{a}_{1}, \ldots, \bar{a}_{\ell}$ and $k_{1}, \ldots, k_{\ell}$ are invariants of the $G$-graded algebra $0=$ $\bigoplus_{g \in G} \mathcal{O}_{g}$. If the $G$-grading on $\mathcal{O}$ is $\Gamma_{\mathscr{O}}(G, P, \gamma)$, then, up to a permutation, $g_{1} P=$ $\cdots=g_{k_{1}} P=\bar{a}_{1}, g_{k_{1}+1} P=\cdots=g_{k_{1}+k_{2}} P=\bar{a}_{2}$, and so on.

Remark 4.9. Instead of using $\gamma=\left(g_{1}, \ldots, g_{t}\right)$ where some of the cosets $g_{i} P$ may be equal to each other, one can take multiplicities,

$$
\kappa=\left(k_{1}, \ldots, k_{\ell}\right), \quad \text { where } k_{i} \text { are positive integers, }
$$

with $|\kappa|:=k_{1}+\cdots+k_{\ell}=t$,

$$
\gamma=\left(g_{1}, \ldots, g_{\ell}\right), \quad \text { where } g_{i} \in G \text { are such that } g_{i}^{-1} g_{j} \notin P \text { for all } i \neq j,
$$

and write

$$
\Gamma_{\mathscr{O}}(G, P, \kappa, \gamma)=\Gamma_{\mathscr{O}}(G, P, \underbrace{g_{1}, \ldots, g_{1}}_{k_{1} \text { times }}, \ldots, \underbrace{g_{\ell}, \ldots, g_{\ell}}_{k_{\ell} \text { times }}) .
$$

Then $\Gamma_{\mathscr{O}}(G, P, \kappa, \gamma)$ is isomorphic to $\Gamma_{\mathscr{O}}(G, \tilde{P}, \tilde{\kappa}, \tilde{\gamma})$ if and only if $\kappa$ and $\tilde{\kappa}$ have the same number of components $\ell$ and there exists a permutation $\pi$ of the set $\{1, \ldots, \ell\}$ such that $\tilde{k}_{i}=k_{\pi(i)}$ and $\tilde{g}_{i} \equiv g_{\pi(i)}(\bmod P)$ for all $i=1, \ldots, \ell$.

Definition 4.10. Fix $0 \leq s \leq m$. For a multi-index $\alpha \in \mathbb{Z}^{m}$, let

$$
\bar{\alpha}:=\left(\alpha_{1}+p \mathbb{Z}, \ldots, \alpha_{s}+p \mathbb{Z}, \alpha_{s+1}, \ldots, \alpha_{m}\right) \in \mathbb{Z}_{p}^{s} \times \mathbb{Z}^{m-s} .
$$


Define a $\mathbb{Z}_{p}^{s} \times \mathbb{Z}^{m-s}$-grading on $\mathbb{O}=\mathcal{O}(m ; \underline{1})$ by declaring the degree of $1+x_{i}$, $i=1, \ldots, s$, and the degree of $x_{i}, i=s+1, \ldots, m$, to be $\overline{\varepsilon_{i}}$. This is the grading $\Gamma_{\mathscr{O}}(G, P, \gamma)$ where $G=\mathbb{Z}_{p}^{s} \times \mathbb{Z}^{m-s}$ (written additively), $P=\mathbb{Z}_{p}^{s}$, and $\gamma=$ $\left(\overline{\varepsilon_{s+1}}, \ldots, \overline{\varepsilon_{m}}\right)$. Denote this grading by $\Gamma_{\mathscr{O}}(s)$.

Corollary 4.11. Let $\mathrm{O}=\mathrm{O}(m ; 1)$. Then, up to equivalence, there are exactly $m+1$ fine gradings of $\mathcal{O}$. They are $\Gamma_{\mathscr{O}}(s)$ for $s=0, \ldots, m$. The universal group of $\Gamma_{\mathscr{O}}(s)$ is $\mathbb{Z}_{p}^{s} \times \mathbb{Z}^{m-s}$.

Proof. All homogeneous components of $\Gamma_{\mathscr{O}}(s)$ are 1-dimensional, so it is a fine grading. All relations in the grading group $\mathbb{Z}_{p}^{s} \times \mathbb{Z}^{m-s}$ come from the fact that $0 \neq\left(\mathbb{O}_{g}\right)^{p} \subset \mathbb{O}_{e}$ for certain elements $g$. Hence $\mathbb{Z}_{p}^{s} \times \mathbb{Z}^{m-s}$ is the universal group of $\Gamma_{\mathscr{O}}(s)$.

For any abelian group $G$ and a $p$-subgroup $P \subset G$ with a basis $\left\{b_{1}, \ldots, b_{s}\right\}$, any $G$-grading $\Gamma_{\mathscr{O}}\left(G, b_{1}, \ldots, b_{s}, g_{1}, \ldots, g_{m-s}\right)$ is induced from the $\left(\mathbb{Z}_{p}^{s} \times \mathbb{Z}^{m-s}\right)$ grading $\Gamma_{\mathcal{O}}(s)$ by the homomorphism $\mathbb{Z}_{p}^{s} \times \mathbb{Z}^{m-s} \rightarrow G$ defined by

$$
\overline{\varepsilon_{i}} \mapsto b_{i} \text { for } i=1, \ldots, s \quad \text { and } \quad \overline{\varepsilon_{i}} \mapsto g_{i-s} \text { for } i=s+1, \ldots, m .
$$

Hence, up to equivalence, there are no other fine gradings. The gradings $\Gamma_{\mathscr{O}}(s)$ are pairwise nonequivalent, because their universal groups are nonisomorphic.

Definition 4.12. The $G$-grading induced by $\Gamma_{\overparen{O}}\left(G, b_{1}, \ldots, b_{s}, g_{1}, \ldots, g_{t}\right)$ (refer to Definition 4.6) on the Lie algebra $W$ will be denoted by

$$
\Gamma_{W}\left(G, b_{1}, \ldots, b_{s}, g_{1}, \ldots, g_{t}\right) \text { or } \quad \Gamma_{W}(G, P, \gamma) .
$$

Explicitly, we declare the degree of each element to be

$$
\left(1+x_{1}\right)^{\alpha_{1}} \cdots\left(1+x_{s}\right)^{\alpha_{s}} x_{s+1}^{\alpha_{s+1}} \cdots x_{m}^{\alpha_{m}} \partial_{i}, \quad \text { where } \alpha \in \mathbb{Z}^{(m ; 1)}, 1 \leq i \leq m,
$$

$$
b_{1}^{\alpha_{1}-\delta_{i, 1}} \cdots b_{s}^{\alpha_{s}-\delta_{i, s}} g_{1}^{\alpha_{s+1}-\delta_{i, s+1}} \cdots g_{t}^{\alpha_{m}-\delta_{i, m}},
$$

where $\delta_{i, j}$ is the Kronecker delta. In particular, the gradings induced by $\Gamma_{\mathscr{O}}(s)$ (see Definition 4.10) will be denoted by $\Gamma_{W}(s)$.

The following is a generalization of a result in [Demuškin 1970] (see also [Strade 2004, Corollary 7.5.2]) on maximal tori of the restricted Lie algebra $W$, which corresponds to the case when $G$ is an elementary $p$-group.

Theorem 4.13. Let $\mathbb{F}$ be an algebraically closed field of characteristic $p>0$. Let $G$ be an abelian group. Let $W=W(m ; \underline{1})$ over $\mathbb{F}$. Assume $m \geq 3$ if $p=2$ and $m \geq 2$ if $p=3$. Then any grading $W=\bigoplus_{g \in G} W_{g}$ is isomorphic to some $\Gamma_{W}(G, P, \gamma)$ as in Definition 4.12. Two G-gradings, $\Gamma_{W}(G, P, \gamma)$ and $\Gamma_{W}(G, \widetilde{P}, \tilde{\gamma})$, are isomorphic if and only if $P=\widetilde{P}$ and $\gamma \sim \tilde{\gamma}$ (see Definition 4.7).

Proof. Combine Theorem 4.8 and Corollary 4.2. 
Corollary 4.14. Let $W=W(m ; \underline{1})$. Assume $m \geq 3$ if $p=2$ and $m \geq 2$ if $p=3$. Then, up to equivalence, there are exactly $m+1$ fine gradings of $W$. They are $\Gamma_{W}(s)$ for $s=0, \ldots, m$. The universal group of $\Gamma_{W}(s)$ is $\mathbb{Z}_{p}^{s} \times \mathbb{Z}^{m-s}$.

We now turn to special algebras.

Proposition 4.15. In the notation of Proposition 4.4, assume that $0=\bigoplus_{g \in G} \mathbb{O}_{g}$ is an $S$-admissible $G$-grading of degree $g_{0}$. Then the elements $y_{1}, \ldots, y_{m}$ can be chosen in such a way that the degrees $a_{1}, \ldots, a_{m} \in G$ of $1+y_{1}, \ldots, 1+y_{s}, y_{s+1}, \ldots, y_{m}$, respectively, satisfy the equation $g_{0}=a_{1} \cdots a_{m}$.

Proof. Choose elements $y_{1}, \ldots, y_{m}$ as in Proposition 4.4. Let $a_{1}, \ldots, a_{m} \in G$ be the degrees of the elements $1+y_{1}, \ldots, 1+y_{s}, y_{s+1}, \ldots, y_{m}$, respectively. We will adjust $y_{1}, \ldots, y_{m}$ to make $a_{1}, \ldots, a_{m}$ satisfy the above equation.

The form $d y_{1} \wedge \cdots \wedge d y_{m}$ is $G$-homogeneous of degree $a_{0}:=a_{1} \cdots a_{m}$. On the other hand,

$$
d y_{1} \wedge \cdots \wedge d y_{m}=f \omega_{S}, \quad \text { where } f=\operatorname{det}\left(\partial_{j} y_{i}\right) .
$$

Since $\omega_{S}$ is $G$-homogeneous of degree $g_{0}$, we conclude that $f$ is $G$-homogeneous of degree $a_{0} g_{0}^{-1}$. Since $f \notin \mathfrak{M}$, we have $a_{0} g_{0}^{-1} \in P$.

First consider the case $s=m$. Then $a_{0} \in P$ and thus $g_{0} \in P$. Also, the $G$ grading in this case is the eigenspace decomposition of $O$ with respect to a torus $T \subset \operatorname{Der}(0)=W$, where $T$ is isomorphic to the group of additive characters of $P$, so $T$ has rank $s=m$. If $g_{0}=e$, then $\omega_{S}$ is $T$-invariant, so $T \subset \operatorname{Stab}_{W}\left(\omega_{S}\right)=S$, which is a contradiction, because the toral rank of $S=S(m ; \underline{1})$ is less than $m$ (in fact, it is $m-1)$. Therefore, in this case we necessarily have $g_{0} \neq e$. It follows that there exists a basis $\left\{b_{1}, \ldots, b_{m}\right\}$ of $P$ such that $g_{0}=b_{1} \cdots b_{m}$. By Proposition 4.4, we can replace $y_{1}, \ldots, y_{m}$ with $\tilde{y}_{1}, \ldots, \tilde{y}_{m}$ so that $1+\tilde{y}_{i}$ is $G$-homogeneous of degree $b_{i}$ for $i=1, \ldots, m$. The proof in this case is complete.

Now assume that $s<m$. Write $a_{0} g_{0}^{-1}=a_{1}^{\ell_{1}} \cdots a_{s}^{\ell_{s}}$. Set $\tilde{y}_{i}=y_{i}$ for $i<m$ and

$$
\tilde{y}_{m}=y_{m}\left(1+y_{1}\right)^{-\ell_{1}} \cdots\left(1+y_{s}\right)^{-\ell_{s}} .
$$

Then $\tilde{y}_{m}$ is $G$-homogeneous of degree $\tilde{a}_{m}=a_{m} a_{1}^{-\ell_{1}} \ldots a_{s}^{-\ell_{s}}$ and hence $\tilde{y}_{1}, \ldots, \tilde{y}_{m}$ are as desired.

In Definition 4.6 of $\Gamma_{\mathscr{O}}(G, P, \gamma)$, we had to choose a basis $\left\{b_{1}, \ldots, b_{s}\right\}$ for $P$. The isomorphism class, that is, the Aut(O)-orbit, of the grading does not depend on this choice. Clearly, the grading is $S$-admissible of degree $g_{0}=b_{1} \cdots b_{s} g_{1} \cdots g_{t}$ and hence it induces a $G$-grading on the Lie algebra $S$ and its derived subalgebras. Let $L=S(m ; \underline{1})^{(1)}$ if $m \geq 3$ and $L=S(m ; \underline{1})^{(2)}$ if $m=2$. Since $g_{0}$ is $\operatorname{Aut}_{S}(\mathrm{O})$-invariant, the induced gradings on $L$ corresponding to different values of $g_{0}$ are not isomorphic. Conversely, suppose $\left\{\tilde{b}_{1}, \ldots, \tilde{b}_{s}\right\}$ is another basis of $P$ such that $\tilde{b}_{1} \cdots \tilde{b}_{s}=b_{1} \cdots b_{s}$ (that is, this basis leads to the same value of $g_{0}$ ). 
Write $\tilde{b}_{j}=\prod_{i=1}^{s} b_{i}^{\alpha_{i j}}$ where $\left(\alpha_{i j}\right)$ is a nondegenerate matrix with entries in the field $G F_{p}$. Set

$$
\tilde{x}_{j}:=\prod_{i=1}^{s}\left(1+x_{i}\right)^{\alpha_{i j}}-1 \quad \text { for } j=1, \ldots, s,
$$

and $\tilde{x}_{j}=x_{j}$ for $j=s+1, \ldots, m$. Then $\tilde{x}_{1}, \ldots, \tilde{x}_{m}$ form a basis of $\mathfrak{M}$ modulo $\mathfrak{M}^{2}$, and $1+\tilde{x}_{j}$ is homogeneous of degree $\tilde{b}_{j}, j=1, \ldots, s$. One readily computes that

$$
\operatorname{det}\left(\partial_{j} \tilde{x}_{i}\right)=\operatorname{det}\left(\alpha_{i j}\right) \prod_{i=1}^{s}\left(1+x_{i}\right)^{-1+\sum_{j=1}^{s} \alpha_{i j}} .
$$

Now $\tilde{b}_{1} \cdots \tilde{b}_{s}=b_{1} \cdots b_{s}$ means that $\sum_{j=1}^{s} \alpha_{i j}=1$ for all $i$, so $\operatorname{det}\left(\partial_{j} \tilde{x}_{j}\right)$ is in $\mathbb{F}$. Therefore, the automorphism of 0 defined by $x_{i} \mapsto \tilde{x}_{i}, i=1, \ldots, m$, belongs to the subgroup $\operatorname{Aut}_{S}(\mathcal{O})$. We have proved that two $G$-gradings on $L$ arising from the same data $P$ and $\gamma$, but different choices of basis for $P$, are isomorphic if and only if they have the same value of $g_{0}$. This justifies the following:

Definition 4.16. Let $P$ and $\gamma$ be as in Definition 4.6. Let $g_{0} \in G$ be such that

$$
g_{0} g_{1}^{-1} \cdots g_{t}^{-1} \in P \backslash\{e\} .
$$

Select a basis $\left\{b_{1}, \ldots, b_{s}\right\}$ for $P$ such that $g_{0}=b_{1} \cdots b_{s} g_{1} \cdots g_{t}$. The $G$-grading induced by $\Gamma_{\mathscr{O}}\left(G, b_{1}, \ldots, b_{t}, g_{1}, \ldots, g_{s}\right)$ on the Lie algebra $S$ and its derived subalgebras will be denoted by $\Gamma_{S}\left(G, b_{1}, \ldots, b_{t}, g_{1}, \ldots, g_{s}\right)$ or $\Gamma_{S}\left(G, P, \gamma, g_{0}\right)$. In particular, the $\left(\mathbb{Z}_{p}^{s} \times \mathbb{Z}^{m-s}\right)$-grading induced by $\Gamma_{\mathscr{O}}(s)$ (see Definition 4.10 , with $\left\{\overline{\varepsilon_{1}}, \ldots, \overline{\varepsilon_{s}}\right\}$ as a basis for $\left.\mathbb{Z}_{p}^{s}\right)$, will be denoted by $\Gamma_{S}(s)$.

The following is a generalization of a result in [Demuškin 1970] (see also [Strade 2004, Theorem 7.5.5]) on maximal tori of the restricted Lie algebra $C S$, which corresponds to the case when $G$ is an elementary $p$-group.

Theorem 4.17. Let $\mathbb{F}$ be an algebraically closed field of characteristic $p>3$. Let $G$ be an abelian group. Let $L=S(m ; \underline{1})^{(1)}$ if $m \geq 3$ and $L=S(m ; \underline{1})^{(2)}=H(m ; \underline{1})^{(2)}$ if $m=2$ ( a simple Lie algebra over $\mathbb{F}$ ). Then any grading $L=\bigoplus_{g \in G} L_{g}$ is isomorphic to some $\Gamma_{S}\left(G, P, \gamma, g_{0}\right)$ as in Definition 4.16. Two G-gradings, $\Gamma_{S}\left(G, P, \gamma, g_{0}\right)$ and $\Gamma_{S}\left(G, \widetilde{P}, \tilde{\gamma}, \tilde{g}_{0}\right)$, are isomorphic if and only if $P=\widetilde{P}, \gamma \sim \tilde{\gamma}$ (Definition 4.7) and $g_{0}=\tilde{g}_{0}$.

Proof. First we show that the grading $L=\bigoplus_{g \in G} L_{g}$ is isomorphic to some grading $\Gamma_{S}\left(G, P, \gamma, g_{0}\right)$. We can apply Corollary 4.2 to translate this problem to the algebra $\mathcal{O}$. Let $\Gamma^{\prime}: \mathcal{O}=\bigoplus_{g \in G} \mathcal{O}_{g}^{\prime}$ be the $S$-admissible grading on $\mathbb{O}$, of some degree $g_{0} \in G$, that induces the grading $L=\bigoplus_{g \in G} L_{g}$. As usual, let $P=\left\{g \in G \mid \mathbb{O}_{g}^{\prime} \not \subset \mathfrak{M}\right\}$ and let $s$ be the rank of $P$. By Proposition 4.15, there exist elements $y_{1}, \ldots, y_{m} \in \mathfrak{M}$ 
that form a basis of $\mathfrak{M} \bmod \mathfrak{M}^{2}$ and such that $1+y_{i}, i \leq s$, and $y_{i}, i>s$, are $G$ homogeneous of some degrees $a_{i}, i=1, \ldots, m$, where $\left\{a_{1}, \ldots, a_{s}\right\}$ is a basis of $P$ and $g_{0}=a_{1} \cdots a_{m}$. We want to show that there exists an automorphism in $\operatorname{Aut}_{S}(\mathrm{O})$ that sends $\Gamma^{\prime}$ to the grading $\Gamma_{\mathscr{O}}=\Gamma_{\mathscr{O}}\left(G, a_{1}, \ldots, a_{s}, a_{s+1}, \ldots, a_{m}\right)$. Denote the latter grading by $0=\bigoplus_{g \in G} \hat{O}_{g}$. Let $\mu$ be the automorphism of 0 defined by $y_{i} \mapsto x_{i}, i=1, \ldots, m$. Then $\mu$ sends $\Gamma^{\prime}$ to $\Gamma_{\mathcal{O}}$, but $\mu$ may not belong to $\operatorname{Aut}_{S}(\mathcal{O})$. Write $\mu\left(\omega_{S}\right)=f \omega_{S}$ for some $f \in \mathbb{O}$. Now $\mu\left(\omega_{S}\right)$ has degree $g_{0}$ relative to the grading induced on $\Omega^{m}$ by $\Gamma_{\mathscr{O}}, \omega_{S}$ has degree $a_{1} \cdots a_{m}$ relative to the said grading, and $g_{0}=a_{1} \cdots a_{m}$, so we conclude that $f$ has degree $e$ relative to $\Gamma_{\mathscr{O}}$. If $s=m$, this implies that $f$ is in $\mathbb{F}$ and hence $\mu \in \operatorname{Aut}_{S}(\mathcal{O})$, completing the proof. So we assume $s<m$.

Now we follow the idea of the proof of [Strade 2004, Proposition 7.5.4], which is due to [Kuznetsov and Yakovlev 1997]. Observe that

$$
\begin{aligned}
\mu\left(\omega_{S}\right) & =\mu\left(d\left(x_{1} d x_{2} \wedge \cdots \wedge d x_{m}\right)\right) \\
& =d\left(\mu\left(x_{1}\right) d \mu\left(x_{2}\right) \wedge \cdots \wedge d \mu\left(x_{m}\right)\right) \\
& =d\left(\sum_{i=1}^{m}(-1)^{i-1} h_{i} d x_{1} \wedge \cdots \wedge d x_{i-1} \wedge d x_{i+1} \wedge \cdots \wedge d x_{m}\right)=\left(\sum_{i=1}^{m} \partial_{i} h_{i}\right) \omega_{S},
\end{aligned}
$$

where $h_{1}, \ldots, h_{m} \in \mathbb{O}$. Set $E:=\sum_{i=1}^{m} h_{i} \partial_{i} \in W$. Since $\mu\left(\omega_{S}\right)=f \omega_{S}$, we have $\operatorname{div}(E)=\sum_{i=1}^{m} \partial_{i} h_{i}=f$. One can immediately verify that $\operatorname{div}\left(W_{g}\right) \subset \mathrm{O}_{g}$ for all $g \in G$, where $\Gamma_{W}: W=\bigoplus_{g \in G} W_{g}$ is the grading induced on $W$ by $\Gamma_{\mathcal{O}}$. (Also, this is a consequence of the fact that div $: W \rightarrow \mathbb{O}$ is $\operatorname{Aut}(\mathcal{O})$-equivariant.) Since $f \in \mathscr{O}_{e}$, replacing $E$ with its $G$-homogeneous component of degree $e$ will not affect the equation $\operatorname{div}(E)=f$, so we will assume that $E \in W_{e}$.

Define a $\mathbb{Z}$-grading on 0 by declaring the degree of $x_{1}, \ldots, x_{s}$ (or, equivalently, $\left.1+x_{1}, \ldots, 1+x_{s}\right)$ to be 0 and the degree of $x_{s+1}, \ldots, x_{m}$ to be 1 . This $\mathbb{Z}$-grading is compatible with the $G$-grading $\Gamma_{\odot}$ in the sense that the homogeneous components of one grading are graded subspaces of 0 relative to the other grading. Denote the filtration associated to this $\mathbb{Z}$-grading by $\mathcal{O}_{\{\ell\}}, \ell=0,1,2, \ldots$, to distinguish it from the filtration $\mathbb{O}_{(\ell)}$ associated to the canonical $\mathbb{Z}$-grading.

Write $f=\sum_{k \geq 0} f_{k}$, where $f_{k}$ has degree $k$ in the $\mathbb{Z}$-grading and degree $e$ in the $G$-grading. Observe that the constant term of $f$ is equal to the constant term of $f_{0}$, so $f_{0}$ is an invertible element of 0 . Let $\tau_{1}$ be the automorphism of 0 defined by

$$
\tau_{1}\left(x_{i}\right)=x_{i} \text { for } i<m \quad \text { and } \quad \tau_{1}\left(x_{m}\right)=f_{0}^{-1} x_{m} .
$$

Since $f_{0}$ has degree $e$ in the $G$-grading, $\tau_{1}$ preserves $\Gamma_{\mathscr{O}}$, that is, $\tau_{1}\left(\mathscr{O}_{g}\right)=\mathscr{O}_{g}$ for all $g \in G$. We also have $\tau_{1}\left(\mathcal{O}_{\{\ell\}}\right)=\mathscr{O}_{\{\ell\}}$ for all $\ell$. Since $x_{m}$ has degree 1 in the 
$\mathbb{Z}$-grading, it does not occur in $f_{0}$. Hence $\tau_{1}\left(f_{0}\right)=f_{0}$ and

$$
\begin{aligned}
\left(\tau_{1} \circ \mu\right)\left(\omega_{S}\right) & =\tau_{1}\left(f \omega_{S}\right)=\tau_{1}(f) \tau_{1}\left(\omega_{S}\right) \\
& =\left(f_{0}+\tau_{1}(\tilde{h})\right) f_{0}^{-1} \omega_{S}=(1+h) \omega_{S},
\end{aligned}
$$

where $\tilde{h}=\sum_{k \geq 1} f_{k}$ and $h=f_{0}^{-1} \tau_{1}(\tilde{h})$. Note that $h \in \mathcal{O}_{\{1\}}$.

Claim. For any $\ell=1,2, \ldots$ there exists an automorphism $\tau_{\ell}$ of $\bigcirc$ that preserves the $G$-grading $\Gamma_{0}$ and satisfies

$$
\left(\tau_{\ell} \circ \mu\right)\left(\omega_{S}\right)=(1+h) \omega_{S}, \quad \text { where } h \in \mathcal{O}_{\{\ell\}} .
$$

We proceed by induction on $\ell$. The basis for $\ell=1$ was proved above. Assume (35) holds for some $\ell \geq 1$ and $\tau_{\ell}$. Since $\tau_{\ell}$ preserves $\Gamma_{\mathscr{O}}$, we have $1+h \in \mathbb{O}_{e}$ and hence $h \in \mathbb{O}_{e}$. Write $h=\sum_{k \geq \ell} h_{k}$, where $h_{k}$ has degree $k$ in the $\mathbb{Z}$-grading and degree $e$ in the $G$-grading. As was shown above, there exists $E \in W_{e}$ such that $\operatorname{div}(E)=1+h$. Write $E=\sum_{k \geq-1} E_{k}$ where $E_{k}$ has degree $k$ in the $\mathbb{Z}$-grading induced from our $\mathbb{Z}$-grading of $\mathcal{O}$ and degree $e$ in the $G$-grading. Since div preserves the $\mathbb{Z}$-grading, we have $\operatorname{div} E_{k}=h_{k}$ for $k \geq 1$. Let $\tilde{\tau}$ be the automorphism of 0 defined by

$$
\tilde{\tau}\left(x_{i}\right)=x_{i}-E_{\ell}\left(x_{i}\right) \quad \text { for } i=1, \ldots, m .
$$

Since $E_{\ell} \in W_{e}$, the automorphism $\tilde{\tau}$ preserves the $G$-grading $\Gamma_{0}$. We also have $\tilde{\tau}(f)=f\left(\bmod \mathcal{O}_{\{k+1\}}\right)$ for all $f \in \mathbb{O}_{\{k\}}$ and

$$
\tilde{\tau}\left(\omega_{S}\right)=\left(1-\operatorname{div}\left(E_{\ell}\right)+\tilde{f}\right) \omega_{S}=\left(1-h_{\ell}+\tilde{f}\right) \omega_{S}
$$

for some $\tilde{f} \in \mathcal{O}_{\{2 \ell\}}$. Hence

$$
\begin{aligned}
\left(\tilde{\tau} \circ \tau_{\ell} \circ \mu\right)\left(\omega_{S}\right) & =\tilde{\tau}\left((1+h) \omega_{S}\right)=\tilde{\tau}(1+h) \tilde{\tau}\left(\omega_{S}\right) \\
& =\left(1+h_{\ell}+\hat{f}\right)\left(1-h_{\ell}+\tilde{f}\right) \omega_{S}=(1+\tilde{h}) \omega_{S},
\end{aligned}
$$

where $\hat{f} \in \mathcal{O}_{\{\ell+1\}}$ and $\tilde{h}=-h_{\ell}^{2}+\hat{f}\left(1-h_{\ell}\right)+\tilde{f}\left(1+h_{\ell}+\hat{f}\right) \in \mathcal{O}_{\{\ell+1\}}$. Setting $\tau_{\ell+1}=\tilde{\tau} \circ \tau_{\ell}$, we complete the induction step.

Set $\tilde{\mu}=\tau_{\ell} \circ \mu$ for $\ell=(p-1)(m-s)+1$. Then $\tilde{\mu}$ sends $\Gamma^{\prime}$ to $\Gamma_{\overparen{O}}$ and belongs to $\operatorname{Aut}_{S}(\mathcal{O})$, since $\tilde{\mu}\left(\omega_{S}\right)=\omega_{S}$. This proves the first assertion of the theorem.

The subgroup $P$ and the equivalence class of $\gamma=\left(g_{1}, \ldots, g_{t}\right)$ are invariants of the $G$-graded algebra $\mathbb{O}$, and $g_{0}$ is $\operatorname{Aut}_{S}(\mathbb{O})$-invariant. It remains to show that, if $\gamma \sim \tilde{\gamma}$ and $b_{1} \cdots b_{s} g_{1} \cdots g_{t}=g_{0}=\tilde{b}_{1} \cdots \tilde{b}_{s} \tilde{g}_{1} \cdots \tilde{g}_{t}$, where $\left\{b_{1}, \ldots, b_{s}\right\}$ and $\left\{\tilde{b}_{1}, \ldots, \tilde{b}_{s}\right\}$ are bases of $P$ as in Definition 4.16 , then $\left(G, b_{1}, \ldots, b_{s}, g_{1}, \ldots, g_{t}\right)$ and $\left(G, \tilde{b}_{1}, \ldots, \tilde{b}_{s}, \tilde{g}_{1}, \ldots, \tilde{g}_{t}\right)$ are in the same $\operatorname{Aut}_{S}(\mathcal{O})$-orbit. Clearly, the automorphism (31) of 0 , determined by a permutation $\pi$ of $\{1, \ldots, t\}$, belongs to $\operatorname{Aut}_{S}(\mathbb{O})$. So it suffices to consider the case $\tilde{g}_{i} \equiv g_{i}(\bmod P)$. Write $\tilde{b}_{j}=\prod_{i=1}^{s} b_{i}^{\alpha_{i j}}$, where $\left(\alpha_{i j}\right)$ is a nondegenerate matrix with entries in the field $G F_{p}$. Also write 
$\tilde{g}_{i}=g_{i} \prod_{j=1}^{s} b_{j}^{\ell_{i j}}$ for $i=1, \ldots, t$. Then the composition $\mu$ of the automorphism defined by $x_{j} \mapsto \tilde{x}_{j}, j \leq s$, and $x_{j} \mapsto x_{j}, j>s$, where $\tilde{x}_{j}$ are as in (33), and the automorphism defined by (32) sends $\Gamma_{\mathscr{O}}\left(G, \tilde{b}_{1}, \ldots, \tilde{b}_{s}, \tilde{g}_{1}, \ldots, \tilde{g}_{t}\right)$ to $\Gamma_{\mathscr{O}}\left(G, b_{1}, \ldots, b_{s}, g_{1}, \ldots, g_{t}\right)$. Now, (34) implies that

$$
\begin{aligned}
\mu\left(\omega_{S}\right) & =\operatorname{det}\left(\alpha_{i j}\right)\left(\prod_{i=1}^{s}\left(1+x_{i}\right)^{-1+\sum_{j=1}^{s} \alpha_{i j}}\right)\left(\prod_{i=1}^{t} \prod_{j=1}^{s}\left(1+x_{j}\right)^{\ell_{i j}}\right) \omega_{S} \\
& =\operatorname{det}\left(\alpha_{i j}\right)\left(\prod_{i=1}^{s}\left(1+x_{i}\right)^{-1+\sum_{j=1}^{s} \alpha_{i j}+\sum_{j=1}^{t} \ell_{j i}}\right) \omega_{S} .
\end{aligned}
$$

On the other hand,

$$
\tilde{b}_{1} \cdots \tilde{b}_{s} \tilde{g}_{1} \cdots \tilde{g}_{t}=g_{1} \cdots g_{t} \prod_{i=1}^{s} b_{i}^{\sum_{j=1}^{s} \alpha_{i j}+\sum_{j=1}^{t} \ell_{j i}},
$$

so the equality $\tilde{b}_{1} \cdots \tilde{b}_{s} \tilde{g}_{1} \cdots \tilde{g}_{t}=g_{0}=b_{1} \cdots b_{s} g_{1} \cdots g_{t}$ implies that

$$
\sum_{j=1}^{s} \alpha_{i j}+\sum_{j=1}^{t} \ell_{j i}=1 \text { for all } i
$$

and hence $\mu \in \operatorname{Aut}_{S}(\mathbb{O})$.

Corollary 4.18. Under the assumptions of Theorem 4.17, there are, up to equivalence, exactly $m+1$ fine gradings of $L$. They are $\Gamma_{S}(s)$ for $s=0, \ldots, m$. The universal group of $\Gamma_{S}(s)$ is $\mathbb{Z}_{p}^{s} \times \mathbb{Z}^{m-s}$.

\section{References}

[Allen and Sweedler 1969] H. P. Allen and M. E. Sweedler, "A theory of linear descent based upon Hopf algebraic techniques”, J. Algebra 12:2 (1969), 242-294. MR 39 \#4233 Zbl 0257.16024

[Bahturin and Kochetov 2010] Y. Bahturin and M. Kochetov, "Classification of group gradings on simple Lie algebras of types $\mathscr{A}, \mathscr{B}, \mathscr{C}$ and $\mathscr{D}$ ", J. Algebra 324:11 (2010), 2971-2989. MR 2732982 Zbl 05853355

[Demuškin 1970] S. P. Demuškin, "Cartan subalgebras of the simple Lie $p$-algebras $W_{n}$ and $S_{n}$ ", Sibirsk. Mat. Ž. 11 (1970), 310-325. In Russian. MR 41 \#6919

[Dieudonné 1973] J. Dieudonné, Introduction to the theory of formal groups, Pure and Applied Math. 20, Dekker, New York, 1973. MR 48 \#11128 Zbl 0287.14013

[Elduque 2010] A. Elduque, "Fine gradings on simple classical Lie algebras", J. Algebra 324:12 (2010), 3532-3571. MR 2011j:17049 Zbl 1213.17030

[Kochetov 2009] M. Kochetov, "Gradings on finite-dimensional simple Lie algebras", Acta Appl. Math. 108:1 (2009), 101-127. MR 2010i:17044 Zbl 05644664

[Kuznetsov and Yakovlev 1997] M. I. Kuznetsov and V. A. Yakovlev, "Elementary proof of Demuskin's theorem in tori in special Lie p-algebras of Cartan type", Comm. Algebra 25:12 (1997), 3979-3983. MR 1481581 Zbl 0904.17018 
[Montgomery 1993] S. Montgomery, Hopf algebras and their actions on rings, CBMS Regional Conf. Ser. in Math. 82, Amer. Math. Soc., Providence, RI, 1993. MR 94i:16019 Zbl 0793.16029

[Skryabin 1995] S. M. Skryabin, "Modular Lie algebras of Cartan type over algebraically non-closed fields, II", Comm. Algebra 23:4 (1995), 1403-1453. MR 96a:17013 Zbl 0857.17017

[Skryabin 2001] S. Skryabin, "On the automorphism group schemes of Lie algebras of Witt type", Comm. Algebra 29:9 (2001), 4047-4077. MR 2002k:17021 Zbl 1028.17013

[Strade 2004] H. Strade, Simple Lie algebras over fields of positive characteristic, I: Structure theory, de Gruyter Exp. in Math. 38, de Gruyter, Berlin, 2004. MR 2005c:17025 Zbl 1074.17005

[Sweedler 1967] M. E. Sweedler, "Hopf algebras with one grouplike element", Trans. Amer. Math. Soc. 127:3 (1967), 515-526. MR 35 \#1634 Zbl 0168.26901

[Waterhouse 1971] W. C. Waterhouse, "Automorphism schemes and forms of Witt Lie algebras", J. Algebra 17 (1971), 34-40. MR 43 \#305 Zbl 0212.25602

[Waterhouse 1979] W. C. Waterhouse, Introduction to affine group schemes, Graduate Texts in Math. 66, Springer, New York, 1979. MR 82e:14003 Zbl 0442.14017

[Waterhouse 1991] W. C. Waterhouse, "Automorphisms and twisted forms of generalized Witt Lie algebras”, Trans. Amer. Math. Soc. 327:1 (1991), 185-200. MR 91m:17025 Zbl 0733.17007

Received July 28, 2010.

YURI BAHTURIN

DEPARTMENT OF MATHEMATICS AND STATISTICS

MEMORIAL UNIVERSITY OF NEWFOUNDLAND

ST. JOHN'S, NL A1C 5S7

CANADA

bahturin@mun.ca

http://www.math.mun.ca/ yuri/

MikHaIl Kochetov

DEPARTMENT OF MATHEMATICS AND STATistics

MEMORIAL UNIVERSITY OF NEWFOUNDLAND

ST. JOHN's, NL A1C 5S7

CANADA

mikhail@mun.ca

http://www.math.mun.ca/ mikhail/ 


\title{
PACIFIC JOURNAL OF MATHEMATICS
}

\author{
http://pacificmath.org \\ Founded in 1951 by \\ E. F. Beckenbach (1906-1982) and F. Wolf (1904-1989)
}

\section{EDITORS}

V. S. Varadarajan (Managing Editor)

Department of Mathematics

University of California

Los Angeles, CA 90095-1555

pacific@math.ucla.edu

Vyjayanthi Chari

Department of Mathematics

University of California

Riverside, CA 92521-0135

chari@math.ucr.edu

\section{Robert Finn}

Department of Mathematics Stanford University

Stanford, CA 94305-2125

finn@math.stanford.edu

Kefeng Liu

Department of Mathematics

University of California

Los Angeles, CA 90095-1555

liu@math.ucla.edu
Darren Long

Department of Mathematics

University of California

Santa Barbara, CA 93106-3080

long@math.ucsb.edu

Jiang-Hua Lu

Department of Mathematics

The University of Hong Kong

Pokfulam Rd., Hong Kong jhlu@maths.hku.hk

Alexander Merkurjev

Department of Mathematics

University of California

Los Angeles, CA 90095-1555

merkurev@math.ucla.edu
Sorin Popa

Department of Mathematics University of California

Los Angeles, CA 90095-1555 popa@math.ucla.edu

Jie Qing

Department of Mathematics

University of California

Santa Cruz, CA 95064

qing@cats.ucsc.edu

Jonathan Rogawski

Department of Mathematics

University of California

Los Angeles, CA 90095-1555

jonr@math.ucla.edu

\section{PRODUCTION}

pacific@math.berkeley.edu

\section{SUPPORTING INSTITUTIONS}

ACADEMIA SINICA, TAIPEI

CALIFORNIA INST. OF TECHNOLOGY INST. DE MATEMÁTICA PURA E APLICADA KEIO UNIVERSITY

MATH. SCIENCES RESEARCH INSTITUTE NEW MEXICO STATE UNIV.

OREGON STATE UNIV.

\author{
STANFORD UNIVERSITY \\ UNIV. OF BRITISH COLUMBIA \\ UNIV. OF CALIFORNIA, BERKELEY \\ UNIV. OF CALIFORNIA, DAVIS \\ UNIV. OF CALIFORNIA, LOS ANGELES \\ UNIV. OF CALIFORNIA, RIVERSIDE \\ UNIV. OF CALIFORNIA, SAN DIEGO \\ UNIV. OF CALIF., SANTA BARBARA
}

\author{
UNIV. OF CALIF., SANTA CRUZ \\ UNIV. OF MONTANA \\ UNIV. OF OREGON \\ UNIV. OF SOUTHERN CALIFORNIA \\ UNIV. OF UTAH \\ UNIV. OF WASHINGTON \\ WASHINGTON STATE UNIVERSITY
}

These supporting institutions contribute to the cost of publication of this Journal, but they are not owners or publishers and have no responsibility for its contents or policies.

See inside back cover or pacificmath.org for submission instructions.

The subscription price for 2011 is US \$420/year for the electronic version, and \$485/year for print and electronic.

Subscriptions, requests for back issues from the last three years and changes of subscribers address should be sent to Pacific Journal of Mathematics, P.O. Box 4163, Berkeley, CA 94704-0163, U.S.A. Prior back issues are obtainable from Periodicals Service Company, 11 Main Street, Germantown, NY 12526-5635. The Pacific Journal of Mathematics is indexed by Mathematical Reviews, Zentralblatt MATH, PASCAL CNRS Index, Referativnyi Zhurnal, Current Mathematical Publications and the Science Citation Index.

The Pacific Journal of Mathematics (ISSN 0030-8730) at the University of California, c/o Department of Mathematics, 969 Evans Hall, Berkeley, CA 94720-3840, is published monthly except July and August. Periodical rate postage paid at Berkeley, CA 94704, and additional mailing offices. POSTMASTER: send address changes to Pacific Journal of Mathematics, P.O. Box 4163, Berkeley, CA 94704-0163.

PJM peer review and production are managed by EditFLOW ${ }^{\mathrm{TM}}$ from Mathematical Sciences Publishers.

PUBLISHED BY PACIFIC JOURNAL OF MATHEMATICS

at the University of California, Berkeley 94720-3840

A NON-PROFIT CORPORATION

Typeset in LATEX

Copyright $(2011$ by Pacific Journal of Mathematics 


\section{PACIFIC JOURNAL OF MATHEMATICS}

Volume $253 \quad$ No. $2 \quad$ October 2011

Fusion rules on a parametrized series of graphs

MARTA AsAeda and UfFE HAAGERUP

Group gradings on restricted Cartan-type Lie algebras

YURI BAHTURIN and MIKHAIL KOCHETOV

B2-convexity implies strong and weak lower semicontinuity of partitions of $\mathbb{R}^{n}$

\section{DAVID G. CARABALLO}

Testing the functional equation of a high-degree Euler product

DAVID W. FARMER, NATHAN C. RYAN and RALF SCHMIDT

Asymptotic structure of a Leray solution to the Navier-Stokes flow around a 367 rotating body

Reinhard Farwig, Giovanni P. Galdi and Mads Kyed

Type II almost-homogeneous manifolds of cohomogeneity one

DANIEL GUAN

Cell decompositions of Teichmüller spaces of surfaces with boundary

REN GUO and FENG LUO

A system of third-order differential operators conformally invariant under $\mathfrak{s l}(3, \mathbb{C})$ and $\mathfrak{s o}(8, \mathbb{C})$

Toshinis A KUBO

Axial symmetry and regularity of solutions to an integral equation in a half-space

GUOZHEN LU and JIUYi ZHU

Braiding knots in contact 3-manifolds

\section{ELENA PAVELESCU}

Gradient estimates for positive solutions of the heat equation under geometric flow

JUN SUN 